Astrophysical Journal, Submitted

Preprint typeset using LATEX style emulateapj v. 08/22/09

\title{
CHANDRA'S VIEW OF THE RAM PRESSURE STRIPPED GALAXY M86
}

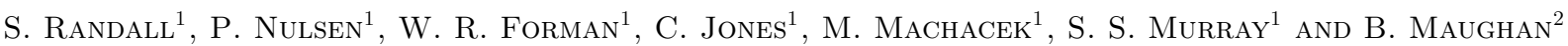 \\ Astrophysical Journal, submitted
}

\begin{abstract}
We present results from a mosaic of nine Chandra observations of M86 and the surrounding field. We detect three main diffuse components: the Virgo ICM at $\sim 2.4 \mathrm{keV}$, the extended halo of M86 at $\sim 1.2 \mathrm{keV}$, and the cooler central and stripped gas of M86 at $\sim 0.8 \mathrm{keV}$. The most striking feature is a long tail of emission, which consists of a plume $\sim 4^{\prime}$ north of M86 and two main extensions emanating from the plume. Based on the morphology and temperature structure of the tail, we conclude that it is formed by ram pressure stripping of M86 as it falls into the Virgo cluster and interacts with the Virgo ICM, in agreement with earlier work. The tail is $150 \mathrm{kpc}$ in projection, and a simple estimate gives a lower limit on the true length of the tail of $380 \mathrm{kpc}$, making this the longest ram pressure stripped tail presently known. The total gas mass in the plume $\left(\sim 7 \times 10^{8} \mathrm{M}_{\odot}\right)$ and tail $\left(\sim 1 \times 10^{9} \mathrm{M}_{\odot}\right)$ is about three times that in the core of M86, which supports the scenario where most of the gas was stripped rapidly and recently. The projected position of the plume can be understood if M86 has an aspherical potential, as suggested by optical isophotes. Ram pressure stripping from an aspherical potential can also explain the split "double tails" seen in M86 and in other Virgo cluster galaxies in the field. The large line-of-sight velocity of M86 (1550 km s${ }^{-1}$ with respect to M87), its position relative to the Virgo cluster, and the orientation of the tail tightly constrain its orbital parameters. The data are inconsistent with a radial orbit, and imply inner and outer turning radii of $r_{\mathrm{i}} \approx 300 \mathrm{kpc}$ and $r_{\mathrm{o}} \gtrsim 8.8 \mathrm{Mpc}$, indicating that M86 is, at best, only weakly bound to the Virgo cluster.

Subject headings: galaxies: clusters: general — galaxies: clusters: individual (Virgo) — magnetic fields - X-rays: galaxies — galaxies: individual (NGC4406) — galaxies: individual
\end{abstract} (M86)

\section{INTRODUCTION}

M86 (NGC 4406) is a bright elliptical (E3/S0) galaxy in the Virgo cluster of galaxies. It is the dominant member of one of the larger subgroups within Virgo (Binggeli et al. 1993; Böhringer et al. 1994; Schindler et al. 1999). Its line-of-sight velocity relative to M87, the dominant member of the Virgo cluster, is $-1550 \mathrm{~km} \mathrm{~s}^{-1}$, much higher than the average cluster velocity dispersion (Smith et al. 2000). The X-ray surface brightness distribution is unusual, with a large "plume" extending to the northwest from M86, which was first noticed as part of a survey of Virgo cluster galaxies undertaken with the Einstein Observatory (Forman et al. 1979). The galaxy has an optical asymmetry that extends in a direction similar to the direction of the plume (Nulsen \& Carter 1987; Mihos et al. 2005). Several authors have interpreted this plume as arising from ram pressure stripping due to strong interactions with the Virgo ICM (Forman et al. 1979; Fabian et al. 1980; Takeda et al. 1984; Knapp et al. 1989; Bregman \& Roberts 1990; White et al. 1991; Rangarajan et al. 1995). Elmegreen et al. (2000) find dust streamers in the core of M86 that connect to the nucleated dwarf galaxy VCC 882, and suggest a recent interaction between the pair that may have contributed to the asymmetry of M86's optical isophotes.

At X-ray energies, M86 has been observed by Einstein (Forman et al. 1979; White et al. 1991), Ginga (Takano et al. 1989), EXOSAT (Edge 1990), ASCA (Matsushita

\footnotetext{
${ }^{1}$ Harvard-Smithsonian Center for Astrophysics, 60 Garden St., Cambridge, MA 02138, USA

${ }^{2}$ Astrophysics Group, Bristol University, Tyndall Avenue, Bristol, BS8 1TL, UK
}

et al. 1994), and ROSAT (Böhringer et al. 1994; Rangarajan et al. 1995). Recently, Finoguenov et al. (2004) presented XMM-Newton observations of M86 and concluded that its unusual morphology is due to an interaction with an X-ray filament rather than the Virgo ICM. However, their conclusions were based on the large separation between M86 and M87 along the line of sight of $2.4 \pm 1.4 \mathrm{Mpc}$ reported by Neilsen \& Tsvetanov (2000), which is inconsistent with the more recent result from Mei et al. (2007) who find a separation of $0.4 \pm 0.8 \mathrm{Mpc}$.

We report here on a mosaic of nine Chandra observations of M86 and the surrounding field, totaling $240 \mathrm{ksec}$ of exposure. The observations and data reduction techniques are described in $\S 2$. The X-ray image is presented in $\S 3$, and results on temperature and abundance structure from spectral analysis are given in $\S$ [. In $\S$ [5] we discuss some of the more interesting features of M86, and place constraints on its orbit. In particular, we give new results on the extent of the stripped tail, and measure the density profile of an X-ray brightness edge seen to the southeast. Our results are summarized in $\S 6$,

We assume a distance to the Virgo cluster of $16 \mathrm{Mpc}$ throughout, consistent with the latest results from Mei et al. (2007), which gives a scale of $0.08 \mathrm{kpc} /{ }^{\prime \prime}$ for $\Omega_{0}=0.3$, $\Omega_{\Lambda}=0.7$, and $H_{0}=70 \mathrm{~km} \mathrm{~s}^{-1} \mathrm{Mpc}^{-1}$. All error ranges are $90 \%$ confidence intervals, unless otherwise stated.

\section{OBSERVATIONS AND DATA REDUCTION}

Table 1 summarizes the nine Chandra observations of M86, which completely cover the center of the galaxy, as well as the plume and long tail. All data were reprocessed from the level 1 events files using the latest calibration files (as of CIAO3.3). CTI and time-dependent gain 
corrections were applied where applicable. LC_CLEAN was used to remove background flares ${ }^{3}$. After periods with obvious flares were removed, the mean rate was calculated for some observed quiescent time interval, and the program was re-run, forcing the global mean to equal this quiescent rate. For observations without obvious flares, time bins that were not within $3 \sigma$ of the mean were discarded. The final cleaned exposure times are given in column (5) of Table 1

The emission from M86 and the surrounding Virgo cluster fills the image field of view for each observation. We therefore used the standard $\mathrm{CALDB}^{4}$ blank sky background files appropriate for each observation, normalized to our observations in the 10-12 keV energy band. We combined background maps for each pointing separately, and generated exposure maps to account for the pointing offsets and the different CCD responses. To generate exposure maps, we assumed a MEKAL model with $k T=1 \mathrm{keV}$, Galactic absorption, and abundance of $30 \%$ solar at a redshift $z=0$, which is consistent with typical results from detailed spectral fits (see $\S$ 4).

\section{THE X-RAY IMAGE}

The exposure corrected, background subtracted, smoothed mosaic image is shown in Figure 11 Several patches of diffuse emission are seen in M86, as is an impressive tail extending to the northwest. To enhance the visibility of the diffuse emission we created an image with bright point sources removed. For each Chandra pointing, regions containing point sources were "filled in" using a Poisson distribution whose mean was equal to that of a local annular background region. The resulting smoothed image (Figure 2) shows:

- a long tail extending to the NW, first detected by Forman et al. (1979). The tail is bifurcated at its base, with the brighter (northern) extension at a position angle of $305^{\circ}$ (measured from north to east) and the shorter extension at $285^{\circ}$. At $18.4^{\prime}$ $(88.4 \mathrm{kpc})$ from the center of the bright emission at the base of the tail (or 21.1' (101.5 kpc) from the center of M86), the tail turns directly north for $6.8^{\prime}(32.5 \mathrm{kpc})$. It then curves to the west at $26.7^{\prime}$ (128.1 kpc) from M86, and finally is no longer detected at $30.6^{\prime}(150 \mathrm{kpc})$. There is evidence for a fainter, parallel tail along the northeastern edge of the tip of the bright tail, discussed below.

- a large plume of emission, directly north of M86, at the root of the long tail (noted previously by Forman et al. 1979). The tail emanates from this clump, rather than from M86 itself as one would expect for a continuous stripping process.

- a sharp boundary along the northeastern edge of the tail, and an apparent "void" in the diffuse emission just north of the tail. This is in stark contrast to the southwestern edge of the tail, where the surface brightness falls off smoothly. Rangarajan et al. (1995) first noted this void, and suggested that it is an evacuated Mach cone left from M86's passage through the outer regions of the Virgo ICM.

3 http://asc.harvard.edu/contrib/maxim/acisbg/

4 http://cxc.harvard.edu/caldb/
- an extended halo of X-ray emission associated with M86 with an edge $13^{\prime}(62.4 \mathrm{kpc})$ to the southeast. This edge also is visible in ROSAT observations (see Figure 3).

- an asymmetry in the diffuse emission near the core of M86, with an extension to the south. Rangarajan et al. (1995) suggest that this is a high pressure region, formed as the leading edge of M86 plows into the Virgo ICM.

- several nearby galaxies. The elliptical galaxy M84, west of M86, shows complicated structure in the diffuse emission in the core related to the radio outburst, and a tail extending to the south (Finoguenov \& Jones 2002). Also visible are NGC 4388 (south-southwest of M86; Beckmann et al. 2004) and NGC 4438 (east of M86; Machacek et al. 2004; Vollmer et al. 2005). Both also have diffuse tails, which likely indicate their projected direction of motion with respect to the Virgo ICM. Most tails split into two main streams. A small patch of faint emission also can be seen in the region of the disk galaxy NGC 4402 (Crowl et al. 2005).

For comparison, the (heavily binned) Chandra mosaic image is plotted alongside the ROSAT and DSS images in Figure 3. The ROSAT image clearly shows the distribution of diffuse emission on larger scales. In particular, the contribution from the M87 halo is seen in the southeast, as is a large halo of diffuse emission around M86 itself, outside the brightest central regions. These components are individually analyzed in our detailed spectral fits (see $\S 4.2$ ). Results from these fits indicate that the extended M86 halo is composed of group gas associated with M86, at a temperature of $k T \approx 1.2 \mathrm{keV}$.

A close up view of the stripped tail, with scaling and binning chosen to show its structure more clearly, is presented in Figure 4. The twisting and curving of the tail at the faint tip may be caused by turbulence or similar "weather" in the Virgo ICM. The tail is clearly bifurcated at its base. Additionally, a fainter tail, split from the first, is barely visible along the northern edge of the faint end of the main tail. To test the significance of this feature, we extracted the total count rate in evenly spaced bins across the width of the box shown in Figure 4. Figure 5 shows two distinct peaks. If we take an average count rate from the highest three bins in each peak and compare it with the average count rate from the lowest two bins in the gap, we find that the gap is significant at about $2.5 \sigma$. As described below in $\S 5.4$. these double streams, as seen clearly at the base of the tail and possibly at the faint end of the tail, as well as in other Virgo cluster galaxies in the field, may be explained as stripping from an inclined aspherical potential.

\section{SPECTRAL ANALYSIS}

In summary, the X-ray images show three diffuse emission components: the Virgo ICM (centered on M87), the extended M86 halo, and the core and tail of M86. We generate a temperature map as a guide for detailed spectral fitting to disentangle the various components. We assume a galactic absorption of $N_{H}=2.62 \times 10^{20} \mathrm{~cm}^{-2}$ 
throughout. Allowing the absorption to vary did not significantly improve any of the spectral fits.

\subsection{Temperature Map}

The temperature map was derived using a method employed by O'Sullivan et al. (2005) and Maughan et al. (2006). For each temperature map pixel (19.7" / pix), we extracted a spectrum from a circular region containing 1000 net counts (after subtracting the blank sky background and a component to account for emission from M87, see $\S$ 4.2), up to a maximum radius of $3.3^{\prime}$ (on the order of the chip size). This large maximum radius was used to obtain sufficient source counts to extend the temperature map to faint regions of the tail. The resulting spectrum was fit in the $0.6-2.0 \mathrm{keV}$ range with an absorbed APEC model using XSPEC. Data from backside- (BI) and front-side-illuminated (FI) CCDs were treated as separate data groups for each observation. For each fit, the abundance was fixed at $26 \%$ solar (consistent with most of the detailed spectral fits; see Table 2). The resulting temperature map is shown in Figure 6 (middle). Regions that did not have at least 1000 source counts within the maximum $3.3^{\prime}$ radius were excluded from the map, as were regions in the southeast that were dominated by Virgo cluster emission. For comparison, we also show the tessellated temperature map in Figure 6 . Each bin was fit using only counts from that area, so the extraction regions are well-defined. The bins were generated using the algorithm provided by Diehl \& Statler (2006), which is a generalization of Cappellari \& Copin's (2003) Voronoi binning algorithm, and requiring roughly 1100 net counts per bin. Each bin was fit with a single APEC model, without a component to model the Virgo ICM background, in contrast to the "smoothed" temperature map. The size of the bins roughly indicate the size of the extraction regions for the smoothed temperature map pixels in the same area. The tessellated and smoothed temperature maps are in good agreement, except in the regions far from M86 where the contribution from the Virgo cluster is more important.

In these temperature maps, the remarkably sharp boundary between the cool galaxy gas and that of the cluster on the northern edge of the tail is apparent (Figure 6). At least four distinct cold clumps can be seen: one centered on M86, one $1.41^{\prime}(6.8 \mathrm{kpc})$ south of M86, one elongated clump 2.45' (11.7 kpc) east of M86, and a larger cool plume $3.63^{\prime}(17.4 \mathrm{kpc})$ to the north. Each clump corresponds to a bright peak in the X-ray image. Additionally, the cooler tail extends to the northwest. To the southeast, in the region of the brightness edge discussed in $\S 5.2$, the gas is somewhat hotter $(\sim 0.25 \mathrm{keV})$ in the southern half of this feature than in the northeastern half at the same distance from M86. This temperature difference is detected in spectra from non-overlapping regions in these two areas, indicating that the difference is not an artifact of the technique used to generate the temperature map. More detailed fits are discussed in $\S 4.2$.

\subsection{Detailed Spectra}

Based on the derived temperature map and the X-ray image, we defined 23 regions for detailed spectral analysis (see Figure 77). To include data from a particular observation in the spectral analysis, we required that it contribute at least 200 counts (each data group was binned such that there were at least 40 counts per bin). A summary of the regions and spectral model for each region are given in Table 2, R1 - R4 are centered on the individual clumps of emission near M86, while R5 -R10 trace the long tail NW of M86 (hereafter regions will be labeled as 'R\#', with the number defined by column 1 of Table 2). R11 roughly corresponds to the "shocked region" indicated by Finoguenov et al. (2004; see their figure 7). There are several regions to either side of the M86 tail. R17, R18, R19, \& R23 measure the large scale diffuse components from M86 and M87, outside the central region where the cool gas is observed (note that R23 is a combination of five disjoint regions, see Figure (7). Finally, R14 - R16 contain M84 and its tail of diffuse emission. Each region was initially fit with an absorbed APEC model over the $0.6-5.0 \mathrm{keV}$ range, with an additional APEC component to model emission from the Virgo ICM as in $\S 4.1$ with the abundance allowed to vary. Additional components were added, as needed, to improve the fits (see Table2, For example, a single temperature model gave large residuals at high energies when fit to the regions corresponding to the cores of M86 and M84 (R1 and R14, respectively). Since each of these regions is expected to have a contribution from unresolved X-ray point sources, a power law component was added to the model, which greatly improved the fits (and gave a photon index consistent with $\Gamma \approx 1.5$, as expected, see e.g. Sarazin et al. 2003). For regions where this power law component could not be well-constrained, we fixed the photon index at $\Gamma=1.5$. Nine regions showed an improved fit when another APEC model was added (in such cases the abundances of each component were constrained to vary together). Data from each pointing were treated as separate data groups, with normalizations for each pointing allowed to vary independently. As an example, the two temperature plus power law fit to the spectra for the core of M86 (R1) is shown in Figure 8 .

Each of the three main components of the diffuse emission (the Virgo ICM, M86 group gas, and the cooler central M86 gas) was detectable in some subset of the regions shown in Figure 7. In R13 it was possible to fit all three components simultaneously. The Virgo and M86 halos cover most, if not all of the FOV. It was therefore necessary to include these components when doing detailed spectral fits, even in regions where they could not be modeled directly due to inadequate statistics. To account for the varying Virgo contribution, we took the normalizations of the best fit APEC models to R18 and R23 and linearly interpolated across the field based on the distance from the center of M87. Although this only gives a rough estimate, we note that it does not affect our results significantly. For regions closer to M87 (e.g., R19) the interpolation gives a value very close to that measured from R18, which is an appropriate local background. Similarly, regions near the faint end of the M86 tail (e.g., R10) have normalizations close to that of R23, which again, is an appropriate local background for this area. For most regions of interest, M86 is bright enough that the contribution from Virgo is negligible, and the accuracy of interpolating is therefore sufficient.

A similar approach was used to model the contribution from the halo around M86. Although, in general, the 
normalization of this component decreased with distance from the core of M86, the variation across the field was more complicated than for Virgo, which is expected since this gas is in the process of being stripped from M86. We included a fixed component, with $k T=1.2 \mathrm{keV}$, abundance set equal to that of the main component, and variable normalization, in regions where the M86 halo temperature could not be determined directly (see column 2 of Table 2). The effects of accounting for this component can be seen, for example, by comparing lines $1 \& 2$ in Table 2 Including the hotter $1.2 \mathrm{keV}$ component results in a lower best-fit temperature for the cooler gas, and gives a better statistical fit to the data. In general, our results are consistent with Finoguenov et al. (2004), who derive $\mathrm{X}$-ray temperatures and abundances for the central region, including M86 and the plume from XMM-Newton observations.

We note that for the cores of M86 and M84 (R1 and R14 in Table 2) the abundances are poorly determined, despite the large number of net counts. Using a VAPEC model to allow for non-solar abundance ratios did not improve the fits. The failure of these models may be due to abundance variations within the defined regions. For instance, the abundance map given by Finoguenov et al. (2004) shows significant variation near the core of M86, even on relatively small scales. Additionally, the models do not allow for multiphase gas, although the temperature most likely varies significantly in the cores.

Using the detailed spectra, we can derive the approximate mass in cool gas for the base of the tail (the "plume") and the core of M86. Using the temperature and abundance measured in R4, and assuming a prolate spheroid geometry with major and minor axis lengths of $12.3 \mathrm{kpc}$ and $7.9 \mathrm{kpc}$, we find a density of $n_{\text {plume }} \approx$ $8.2 \times 10^{-3} \mathrm{~cm}^{-3}$ and a mass $M_{\text {plume }} \approx 7.4 \times 10^{8} \mathrm{M}_{\odot}$. Similarly, in the core of M86, we find $n_{\text {core }} \approx 6.2 \times 10^{-3} \mathrm{~cm}^{-3}$ and $M_{\text {core }} \approx 7.4 \times 10^{8} \mathrm{M}_{\odot}$ within a sphere of radius $9.6 \mathrm{kpc}$. Assuming a cylindrical geometry for the tail, and considering only the cool gas components in R5 $\mathrm{R} 9$, we find a typical density of $n_{\text {tail }} \approx 1.5 \times 10^{-3} \mathrm{~cm}^{-3}$ and a total gas mass of $M_{\text {tail }} \approx 1.7 \times 10^{9} \mathrm{M}_{\odot}$. The mass in stripped gas is therefore more than three times the gas mass in the core, which supports the scenario where a significant fraction of the M86 ISM is removed rapidly due to ram pressure stripping (Takeda et al. 1984). Rangarajan et al. (1995) find similar results for the plume and the core. However, we find consistent abundance values in the core and in the plume within the errors, while Rangarajan et al. (1995) find a higher abundance in the plume. This disagreement is likely due to the multiphase model we use, which includes a separate component for the $1.2 \mathrm{keV}$ M86 halo emission.

\section{DISCUSSION}

\subsection{The Ram Pressure Stripped Tail of M86}

The most striking feature in Figure 2 is the long tail of emission extending to the NW from M86. M86 has a line-of-sight velocity of $-244 \pm 5 \mathrm{~km} \mathrm{~s}^{-1}$, while M87's is $1307 \pm 7 \mathrm{~km} \mathrm{~s}^{-1}$ (Smith et al. 2000). Therefore, M86 is traversing the Virgo cluster at $v_{\mathrm{M} 86}>1550 \mathrm{~km} \mathrm{~s}^{-1}$ (about Mach 2 for $k T=3 \mathrm{keV}$ ). The tail naturally forms due to ram pressure stripping of the M86 corona by the Virgo cluster ICM. Finoguenov et al. (2004) suggested that the tail formed due to interactions with a filament rather than the Virgo ICM, though their result was based on older distance measurements that placed M86 outside the virial radius of the Virgo cluster. If we assume that M86 is bound to the Virgo cluster, its large line-of-sight relative velocity allows us to constrain the separation between M86 and M87. Using the M87 mass profile detailed in $\S\left[5.3\right.$, we find that a free-fall velocity of $1500 \mathrm{~km} \mathrm{~s}^{-1}$ corresponds to a separation from M87 of $0.5 \mathrm{Mpc}$ (the projected separation is $0.35 \mathrm{Mpc}$ ). This is an upper-limit, since the total relative velocity must be at least as large as the line-of-sight relative velocity. This separation is consistent with recent surface brightness fluctuation distances of Virgo cluster galaxies, which give a distance between M86 and M87 of $0.4 \pm 0.8$ Mpc (Mei et al. 2007).

We also can place a lower-limit on the length of the long stripped tail using the mass profile of M87 and the line-of-sight velocity. The maximum free-fall velocity of M86 from infinity at the projected separation of $0.35 \mathrm{Mpc}$ is about $1680 \mathrm{~km} \mathrm{~s}^{-1}$. Using this as an upper-limit on its current $3 \mathrm{D}$ velocity, we find that the angle between the direction of motion of M86 and the line-of-sight is $\theta \lesssim 23^{\circ}$. Assuming that the stripped tail is aligned with M86's current direction of motion, and given that the length of the tail in the plane of the sky is $150 \mathrm{kpc}\left(0.51^{\circ}\right)$, we find a lower-limit on the actual length of the tail of $L_{\text {tail }} \gtrsim 380 \mathrm{kpc}$, making this the longest ram pressure stripped tail presently known.

The long stripped tail originates in the plume of diffuse emission located directly north of M86. For a continuous stripping process, one would expect the tail to extend from M86 itself. This separation between M86 and the plume can be explained by rapid recent stripping, in which a significant fraction of the remaining gas in M86 is rapidly stripped when the ram pressure stripping condition is met. A detailed discussion of this process is given in $\S 5.4$

As seen from Figure 7 and Table 2, the stripped tail shows a general trend of cooler gas in and near the plume (with temperatures in the $0.8-0.85 \mathrm{keV}$ range) and warmer gas at the tip (in the $0.9-1.2 \mathrm{keV}$ range). The temperature structure of the tail is consistent with a ram pressure stripping model, where, as M86 falls into the Virgo cluster, the hotter, higher entropy group gas is stripped first due to interactions with the Virgo ICM, followed by the cooler, lower entropy M86 ISM, which is removed rapidly once the stripping condition is met.

\subsection{The Extended M86 Halo}

The Chandra and ROSAT X-ray images (Figure 3) show an extended halo associated with M86, with a sharp brightness edge to the southeast in the direction of M87. Since M86 is traversing the Virgo cluster supersonically (see $\oint$ 5.1) we expect a shock to be driven in the Virgo ICM, possibly producing a brightness edge similar to that seen in the southeast. We attempted to measure the density jump associated with the shock in the following way. We extracted the Chandra $0.5-2.0 \mathrm{keV}$ surface brightness profile of this edge in two roughly equal sectors since the morphology of this feature is irregular in the south (see Figure 3), and since the gas temperature differs in these two regions (see $\S 4.1$ ). The emission measure profile for the northern sector is shown in Figure 9. Distance is measured from the center of curvature of the apparent 
edge, which is $10 \mathrm{kpc}\left(2.16^{\prime}\right)$ east of M86. This profile was fit with a spherical gas density model consisting of two power laws. The free parameters were the normalization, the inner $(\alpha)$ and outer $(\beta)$ slopes, the position of the density discontinuity $\left(r_{\text {break }}\right)$, and the amplitude of the jump $(A)$. We assumed that the gas is isothermal with $k T=1 \mathrm{keV}$ and that the abundance is constant at 30\% solar, consistent with results from spectral fits (see $\S$ 4). For the best fit model (see Figure 9), we find $\alpha=0.49_{-0.71}^{+1.14}, \beta=-0.81_{-0.12}^{+0.14}, r_{\text {break }}=52_{-6}^{+5} \mathrm{kpc}$, and $A=1.3_{-0.4}^{+0.3}$. Similar results are found from the southern sector, but with larger errors. The lack of a well-defined edge is consistent with our findings for the orbit of M86 (see $\S 5.3$. In particular, for all of the likely orbits, M86 is moving to the southeast, such that our lines of sight pass through the Mach cone. To see a sharp edge, our line of sight must be tangent to the shock front.

\subsection{Constraints on the Orbit of M86}

Knowing the orbit of M86 is the key to understanding its interaction with the Virgo cluster. The ram pressure stripped tail reveals the motion of the galaxy on the sky. The length and direction of the tail, together with the large line-of-sight speed of M86, constrain its orbit. The large luminosity of M86 suggests that it dominates the associated in-falling subgroup (Schindler et al. 1999). In the following, we assume M86 is bound to the Virgo cluster. For the purpose of calculation, the gravitational potential of the Virgo cluster is treated as a spherical NFW potential (Navarro, Frenk \& White 1997), with a virial radius of $1.3 \mathrm{Mpc}$ (Evrard, Metzler \& Navarro 1996) and a concentration parameter of 4.5 (Neto et al. 2007 ), appropriate for a $\sim 3 \mathrm{keV}$ cluster. The gravitating mass was normalized to match the total mass within $320 \mathrm{kpc}$ of $4.4 \times 10^{13} \mathrm{M}_{\odot}$ (Schindler et al. 1999; scaled to a distance of $16 \mathrm{Mpc}$ ). For this potential, the escape speed from a radius of $351 \mathrm{kpc}$ (the projected separation of M86 from M87) is $1677 \mathrm{~km} \mathrm{~s}^{-1}$, not much larger than M86's line-of-sight speed of $1550 \mathrm{~km} \mathrm{~s}^{-1}$ relative to M87. The empirical mass distribution of Schindler (1999) gives the same escape speed at $351 \mathrm{kpc}$ as our model potential. We note that the gravitational potential of the Virgo cluster is not well constrained at distances from the cluster center comparable to or greater than its virial radius. Furthermore, the truncated NFW potential is highly simplified, ignoring mass beyond the virial radius, departures from spherical symmetry, and the dynamic state of the cluster due to continuing infall. Since some of the limits we derive here are sensitive to the poorly known potential at large radii, they should be treated as indicative rather than quantitative (outside the context of the model). The material in this section is supplemented by a more detailed discussion in Appendix A.

We consider radial orbits first, as suggested by previous studies (e.g., Forman et al. 1979; White et al. 1991). In order for the gas tail to point away from the cluster center, the galaxy must be inbound. It can easily be shown that the line-of sight velocity, $v_{\text {los }}$, is maximized for some radius $r$ greater than the observed separation $s$ (see Appendix A). For a marginally bound (zero energy) radial orbit in the potential described above, the maximum value of $\left|v_{\text {los }}\right|$ occurs when M86 is close to twice its projected distance from the cluster center, giving $\left|v_{\text {los }}\right|=1205 \mathrm{~km} \mathrm{~s}^{-1}$, less than the observed value of $1550 \mathrm{~km} \mathrm{~s}^{-1}$. Thus, unless M86 is significantly unbound from the Virgo cluster (or the potential is incorrect), its line-of-sight speed is inconsistent with radial and nearly radial orbits.

It is convenient to specify more general orbits in terms of their inner and outer turning radii, $r_{\mathrm{i}}$ and $r_{\mathrm{o}}$, respectively. We constrain the possible orbits of M86 by placing limits on these parameters. Coarse limits can be placed on these radii by considering energy arguments alone. Assuming that M86 is bound to the Virgo cluster, we find $r_{\mathrm{i}} \lesssim 489 \mathrm{kpc}$ and $r_{\mathrm{o}} \gtrsim 3.5 \mathrm{Mpc}$, or $\simeq 2.7$ times the virial radius of the Virgo cluster (see Appendix $\mathrm{A}$ ). These limits can be further restricted by considering the range of possible viewing directions for each point on an orbit. There can be zero, two, or four possible viewing directions that would place M86 at the observed separation from the cluster and give it the observed line-of-sight velocity (see Appendix A). Figure 10 shows a range of marginally bound orbits $\left(r_{\mathrm{o}}=\infty\right)$, with regions that meet these conditions marked in color. The range of an orbit where these conditions are met shrinks as $r_{\mathrm{o}}$ decreases, i.e., as the orbit becomes more tightly bound. For the marginally bound orbits, the full range of $r_{\mathrm{i}}$ for which $v_{\text {los }}$ can attain its observed value is $247<r_{\mathrm{i}}<395$ kpc (the range represented in Figure 10). For more tightly bound orbits, the acceptable range of $r_{\mathrm{i}}$ is reduced.

The lower limit on $r_{\mathrm{o}}$ is increased if we consider the extent of the ram pressure stripped tail. The projected orbit must extend to at least the distance $(\sim 100 \mathrm{kpc})$ that the tail projects beyond M86 in the direction away from the cluster center. Locations on the orbits where the lineof-sight speed can attain $-1550 \mathrm{~km} \mathrm{~s}^{-1}$ and these conditions also are met are shown in red in Figure 10, We see that the range of possible locations for M86 on these orbits is tightly constrained. We repeated this analysis for a number of values for the outer turning radius. The range of potential orbits and locations for M86 shrinks with decreasing $r_{\mathrm{o}}$ and no orbits were found to meet these criteria for $r_{\mathrm{o}} \lesssim 8.2 \mathrm{Mpc}$ (6.3 virial radii).

Figure 11 shows projections onto the sky of the marginally bound orbits (corresponding to the midpoints of the red regions of Figure 10) overlaid on the Chandra $0.5-2 \mathrm{keV}$ image, with M86 at the representative location for each orbit. It is evident that the possible orbits for M86 can be pruned further. For example, the orbit on the lower right in Figure 11 does not reach far enough north to produce the remote part of the gas tail. At the other limit, the position angle of M87 measured from M86 is $116^{\circ}$ (east from north), generally eastward of these orbits. Overdense stripped gas tends to fall towards the cluster center, in that direction, so that some stripped gas can reasonably lie to the east of the orbit. Stripped gas cannot lie west of the orbit. Thus the orbits that cross the region to the east of the tail and north of M86, where there is no sign of stripped gas, are unlikely candidates for the orbit of M86.

Note that the orbits in Figure 11 are not simply related to their inner turning radii. The orbit corresponding to the smallest value of the inner turning radius appears second from the right at the top of the figure. At first, 
orbits for increasing values of $r_{\mathrm{i}}$ lie to the left of this, but at a value of $r_{\mathrm{i}}$ approaching (but less than) $s$, the acceptable viewing direction flips from outward to inward (i.e., the acceptable sign of $a^{\prime}$ changes, see Appendix A). At this point, the projected position of the orbit shifts from leftmost to rightmost at the top of Figure 11. Whereas the apparent location where the orbit passes through the virial radius was moving outward, it now moves inward as $r_{\mathrm{i}}$ increases, coming into the field of view for the largest values of $r_{\mathrm{i}}$ here. This results in disjoint ranges of possible orbits for M86. Thus the marginally bound orbits with inner turning radii close to $r_{\mathrm{i}} \simeq 260 \mathrm{kpc}$ and those with inner turning radii in the range $330 \lesssim r_{\mathrm{i}} \lesssim 380 \mathrm{kpc}$ are best suited to model the orbit of M86.

Applying the same criteria to the more tightly bound orbits yields the collection of possible orbits shown in Figure 12. We have not attempted to be highly selective or exhaustive, since that requires a well defined model for the formation of the gas trail. For values of the outer turning radius in the lower end of the acceptable range, the shrinking range of possible orbits and locations excludes orbits with inner turning radii $r_{\mathrm{i}}>s$. All of the acceptable orbits for M86 are weakly bound to the Virgo cluster, with the most tightly bound having outer turning radii at $\simeq 8.8 \mathrm{Mpc}$. All of the possible locations for M86 lie only a little farther from M87 than M86 does in projection. All are close to the plane of the sky, ranging from $167 \mathrm{kpc}$ closer than M87 to $263 \mathrm{kpc}$ farther than M87 from the Sun at the extremes. M86 must also be close to the pericenter of its orbit. The direction of motion of M86 is close to our line of sight, within $16^{\circ}-23^{\circ}$ of it, for all of the cases illustrated.

M86 is traversing the Virgo cluster supersonically $\left(v_{\text {los }}=-1550 \mathrm{~km} \mathrm{~s}^{-1}\right.$ alone is almost twice the sound speed $c_{\mathrm{s}}$, which is taken to be $850 \mathrm{~km} \mathrm{~s}^{-1}$ ). Therefore, we expect a shock to be driven in the Virgo ICM. For the orbits we derive, the angle of inclination is smaller than the opening angle of the Mach cone $\left(\simeq 33^{\circ}\right)$ and we should not expect shock fronts to be visible on the plane of the sky (cf. Rangarajan et al. 1995). This is illustrated in Figure 13, which shows the marginally bound orbit with $r_{\mathrm{i}}=376 \mathrm{kpc}$. Each circle is drawn centered on a point where M86 was at a time $\delta t$ in the past, with a radius of $c_{\mathrm{s}} \delta t$. This is done at equally spaced times to indicate the shape of the Mach cone. The absence of caustics in Figure 13 shows that we would not see the shock front in projection. (While this statement is accurate, except possibly in the small region where the shock front is highly supersonic, the diagram is schematic. For example, if M86 were coming directly towards the Earth, radii of sections of the Mach cone would be $\left(1-c_{\mathrm{s}}^{2} / v^{2}\right)^{-1 / 2} \simeq 1.20$ larger than drawn.) Our lines of sight through the compressed gas behind the shock are longest where the circles pile up to the southeast of M86, consistent with the enhancement in X-ray surface brightness seen $\sim 3^{\prime}$ southeast of M86 (Figure 13).

\subsection{Displacement of Stripped Gas}

The prominent plume of gas lying $3^{\prime}-4^{\prime}(\sim 16 \mathrm{kpc})$ north of M86, noted previously (e.g., Forman et al. 1979), appears to be physically separated from the dense gas remaining in the galaxy and it is centered some distance east of the orbits illustrated in Figure 12. The re- moval of a significant fraction of the ISM in a single blob is expected from rapid ram pressure stripping (Takeda et al. 1984). A highly simplified model for ram pressure stripping treats the plume as a single particle subject to gravity and drag due to its motion through the surrounding gas. Allowing for buoyancy, the net force on the plume due to gravity is $\left(\rho-\rho_{\mathrm{e}}\right) V \mathbf{g}$, where $\rho$ is its density, $V$ is its volume, $\rho_{\mathrm{e}}$ is the density of the ambient ICM, and $\mathbf{g}$ is the local acceleration due to gravity. The drag force on the plume is $-C_{\mathrm{D}} A \rho_{\mathrm{e}} v \mathbf{v}$, where $C_{\mathrm{D}}$ is the drag coefficient, $A$ is the plume cross section, $\mathbf{v}$ is its velocity, and $v=|\mathbf{v}|$. Thus, the net acceleration of the plume is $\mathbf{a}=\left[1-\rho_{\mathrm{e}} / \rho\right] \mathbf{g}-\left[C_{\mathrm{D}} \rho_{\mathrm{e}} A /(\rho V)\right] v \mathbf{v}$. Two of the main parameters of this model are the density contrast, $\rho_{\mathrm{e}} / \rho$, and the factor $C_{\mathrm{D}} A / V$. For the density of the ICM, $\rho_{\mathrm{e}}$, we use the beta model of Schindler et al. $(1999 ; \beta=0.47$, core radius $=2.7^{\prime}$ ). On the grounds that the density of the plume is likely to be lower now (due to its stripping and ejection from the confining potential of M86), we set its density contrast at the projected radius of M86 to be 18 (cf. $\sim 16$ from the numbers above, see $\S 4.2$ ) and treat the density of the plume as constant. The other factor is determined by treating the plume as a sphere with a constant radius of $10 \mathrm{kpc}$, with $C_{\mathrm{D}}=0.75$. As above, this radius is a little larger than the observed radius. To simulate stripping, a gas blob is placed at the location and velocity of M86 at the time it is released from the cluster virial radius. The orbit of the blob is followed, subject to the gravitational acceleration of the cluster and the moving galaxy.

This model cannot account for the current location of the plume if the gravitational potential of M86 is spherical. The path of the blob is determined by the competition between gravity and drag. If the drag is large, the blob is ejected from M86 early on its orbit, and slows quickly until it is falling towards the cluster center at its terminal speed, and ultimately lies farther away from M86 than observed. Its early ejection also leaves it too far back along the orbit of M86. Reducing the relative significance of the drag causes the blob to be ejected later, bringing it closer to its observed position but not far enough from the orbit in the direction of the cluster center.

This issue can be resolved if the gravitational potential of M86 is aspherical. Consider a small gas blob in an aspherical galaxy moving at an inclined angle through the ICM, illustrated schematically in Figure 14. While ram pressure is insufficient to eject the blob, it is driven to an equilibrium position where ram pressure is balanced by gravity. Since the direction of the gravitational field must oppose the drag, the external flow must be perpendicular to the equipotential surface. The equilibrium position therefore lies at a location away from the axis passing through the center of the galaxy and parallel to the flow (e.g., near the point labeled "equipotential" in Figure 14). As the drag starts to overwhelm gravity and displaces the blob in the direction of the flow, the equipotentials the blob encounters tilt, so that it is subject to a component of the gravitational force directed away from the axis. Because forces in the direction of the flow are nearly balanced, this off-axis component of gravity drives the blob farther away from the axis of the flow. Thus, a blob stripped from such a galaxy tends to emerge away from the axis of the flow. Treating the gas as a fluid, 
interstellar gas that is pushed inward by ram pressure along the leading edge of the galaxy flows out preferentially along the long axis of the potential, in the direction of the weakest gravitational force.

The model for the aspherical gravitational potential of M86 used here has the form $\Phi(\mathbf{r})=F(w)$, where $F(w)=\psi \ln [(1+w / c) /(1+w)] / w$ is a minor modification of the standard NFW form $(\psi \ln [1 /(1+w)] / w$ in the same notation). The extra factor of $1+w / c$, where $c$ is the concentration parameter, makes the total mass converge, avoiding the need to truncate the mass distribution at the virial radius (which would create problems for our fourth order integrator). The coordinate $w=\sqrt{\left(x^{2}+y^{2}\right) / a^{2}+z^{2} / b^{2}+1}$, where $a$ is the NFW potential scale length and $b$ is chosen to give the desired ellipticity. The additional 1 under the square root flattens the potential at small $r$, providing a better model for the gravitational force on an extended gas blob (of size comparable to $a$ ) when it is close to the center of the galaxy. This modification also makes the integrator behave better near the center of the galaxy. The virial radius of M86 was set to $100 \mathrm{kpc}$ and its concentration parameter to 8 , roughly the values expected for a massive galaxy. The normalizing factor, $\psi$, was expressed as $\psi=9 \sigma^{2}$, so that $\sigma$ is a rough measure of the line-ofsight velocity dispersion for M86. The value used below is $\sigma=185 \mathrm{~km} \mathrm{~s}^{-1}$. We note that for the model what matters is the ratio of the drag force on the blob to the binding force of the M86 potential, allowing for a trade off between the choice of potential and the density ratio and size of the blob. As a result, the choice of potential is not very critical.

The two remaining parameters are the axial ratio, $a / b$, and the orientation of M86. Consistent with its S0/E3 classification, optical images show that M86 is highly flattened on scales comparable to the projected distance of the plume (e.g. $a / b \simeq 1.56$ for the isophote with $a \simeq 4.5^{\prime}$ in the deep image of Nulsen \& Carter 1987). Based on the discussion above, the potential of M86 would need to have a long axis pointing roughly northward from our line-of-sight. Therefore, we assume it is oblate, using $a / b=2$. The orientation of M86 is then determined by the direction of its minor axis. From the optical images, this has a position angle of $\simeq 35^{\circ}$ on the sky. However, its tilt with respect to the plane of the sky is unknown. We have set the minor axis to point towards us in the north, at an angle of $45^{\circ}$ from the plane of the sky. This is roughly the orientation that maximizes the transverse displacement of the blob. Figure 15] shows the path of the blob on the sky for this set of model parameters for the orbit with $r_{\mathrm{o}}=9.1 \mathrm{Mpc}$ and $r_{\mathrm{i}}=314 \mathrm{kpc}$. In this model, the blob is currently slower than M86 by $355 \mathrm{~km} \mathrm{~s}^{-1}$ along our line of sight and trails it by $38 \mathrm{kpc}$.

Around the edges of the gas halo in M86, where the shear in the external flow is strong (Figure 14), if the effective viscosity is high, viscous stresses pull the interstellar gas out of the galaxy. Alternatively, if the viscosity is low, shear instabilities mix the interstellar gas with the ICM, also stripping it from the galaxy (Nulsen 1982). This stripping is aided by the low pressure due to the Bernoulli effect around the edges of the galaxy, which tends to pull gas into the path of the flow. This process works around all edges of the inclined galaxy, as seen from the direction of the flow. However, it is expected to be greatest at the leading and trailing edges. The shear in the external flow is expected to be greatest near the leading edge, favoring stripping there. The large ram pressure at the leading edge displaces the edge of the interstellar gas deeper into the potential of the galaxy, pushing it towards the trailing edge of the galaxy, where the pressure is lower. Thus, gas at the trailing edge sits higher in the gravitational potential of the galaxy, favoring its removal from the galaxy. This may account for the apparent double streams of gas seen trailing M86 and several other galaxies in the composite image. "Viscous" stripping from the main body of M86 as well as the plume can explain the broad features of the gas trail, though modeling the finer features, such as the smaller blobs of gas lying to the east of the galaxy, requires a more detailed treatment of the gas dynamics (e.g., numerical simulations) and is beyond the scope of this paper.

The proton mean free path due to Coulomb collisions is given approximately by $\lambda \simeq 150(k T)^{2} n_{-3}^{-1} \mathrm{pc}$, where $k T$ is the gas temperature in $\mathrm{keV}$ and the electron density is $10^{-3} n_{-3} \mathrm{~cm}^{-3}$. In terms of this, the Reynolds number is $\operatorname{Re} \simeq v L /(s \lambda)$, where $v$ is the flow speed, $s$ is the sound speed, and $L$ is a relevant length scale. Taking $v=1550 \mathrm{~km} \mathrm{~s}^{-1}$ and $L=10 \mathrm{kpc}$ gives $R e \simeq 20$ in the $2.4 \mathrm{keV} \mathrm{ICM}$, but $\mathrm{Re} \simeq 3000$ for the gas in the $0.77 \mathrm{keV}$ plume (and interstellar gas) if it is exposed directly to the external flow. These values suggest that the external flow can be largely laminar, while the flow in the cool interstellar gas is relatively turbulent. Further complicating matters, the effective mean free path may be significantly smaller than the Coulomb mean free path (e.g., Schekochinin et al. 2007). The Reynolds number for this flow is therefore not well defined, due to the range in gas temperature and uncertainty in the mean free path.

\section{SUMMARY}

We have argued that the plume and long tail of M86 formed due to ram pressure stripping forces generated as M86 falls into the Virgo cluster. Several studies have found a similar interpretation for the formation of this feature (e.g., Forman et al. 1979; Fabian et al. 1980; Rangarajan et al. 1995, however see Bregman \& Roberts 1990; Finoguenov et al. 2004). We concentrate on these main results:

- the plume and long tail observed in the diffuse emission are created by ram pressure stripping as M86 falls into the Virgo cluster. The tail is $150 \mathrm{kpc}$ in projection (a simple estimate, which assumes free-fall velocity for M86 and an NFW potential for M87, gives a lower-limt on the true length of the tail of $380 \mathrm{kpc}$ ), making this the longest ram pressure stripped tail presently known.

- based on the X-ray spectra, we detect three distinct components associated with the M86/Virgo cluster system: the Virgo ICM, with $k T \sim 2.4 \mathrm{keV}$; the extended halo of M86, with $k T \sim 1.2 \mathrm{keV}$; and the cooler central and stripped gas of M86, with $k T \sim 0.8 \mathrm{keV}$. The temperature structure of the tail is consistent with ram pressure stripping, where the higher entropy M86 halo gas is stripped 
first and deposited in the tip of the tail, and the lower entropy M86 ISM is stripped more recently, constituting the base of the tail and the plume.

- the large line-of-sight velocity of M86, and its position relative to the Virgo cluster, tightly constrain its orbit, especially if it is assumed that the gas tail traces the orbit. In particular, the observations are inconsistent with a radial orbit. We show that M86 is at best only marginally bound to the Virgo cluster, with an inner turning radius on the order of $300 \mathrm{kpc}$ as expected from its recent in-fall. Our best-fitting orbital model requires that M86 be close to M87, less than $167 \mathrm{kpc}$ closer than or $263 \mathrm{kpc}$ farther than M87 along our line of sight, which is consistent with the most recent distance estimates based on surface brightness fluctuations (Mei et al. 2007) which give a line-of-sight separation of $0.4 \pm 0.8 \mathrm{Mpc}$.

- the prominent plume of gas lying $3^{\prime}-4^{\prime}$ north of M86 appears to have been rapidly driven from M86 by ram pressure stripping. The projected position of the plume, which does not lie directly on our best-fit model orbit for M86, can be understood if M86 has an aspherical potential (as suggested by optical isophotes). If M86 moves through the
Virgo ICM at an inclination angle relative to the local flow, the gas at the trailing edge is more easily stripped, thereby displacing the gas from the nominal orbit of M86 itself. This model may also explain the apparent double streams of gas seen trailing M86, as well as those in other Virgo galaxies.

- the apparent brightness edge to the southeast seen in $R O S A T$ observations is also seen in the Chandra images. The edge is well fit with a two power law gas density model, with an abrupt jump in density by a factor of $1.3_{-0.4}^{+0.3}$ at the edge (consistent with no jump). Assuming that this brightness edge is the shock generated as M86 supersonically falls into the Virgo cluster, the lack of a well-defined density jump is consistent with what is expected from our orbital model, which suggests that the orientation of the Mach cone would make it difficult to detect the shock edge.

The financial support for this work was provided by NASA contracts NAS8-39073, NAS8-38248, NAS801130, NAS8-03060, the Chandra Science Center, and the Smithsonian Institution.

\section{REFERENCES}

Beckmann, V., Gehrels, N., Favre, P., Walter, R., Courvoisier, T. J.-L., Petrucci, P.-O., \& Malzac, J. 2004, ApJ, 614, 641

Binggeli, B., Popescu, C. C., \& Tammann, G. A. 1993, A\&AS, 98, 297

Birzan, L., Rafferty, D. A., McNamara, B. R., Wise, M. W., \& Nulsen, P. E. J. 2004, ApJ, 607, 800

Böhringer, H., Briel, U. G., Schwarz R. A., Voges, W., Hartner, G., \& Trümper, J. 1994, Nature, 368, 828

Bregman, J. N., \& Roberts, M. S. 1990, ApJ, 362, 468

Cappellari, M., \& Copin, Y. 2003, MNRAS, 342, 345

Crowl, H. H., Kenney, J. D. P., van Gorkom, J. H., \& Vollmer, B. 2005, AJ, 130, 65

Diehl, S., \& Statler, T. S. 2006, MNRAS, 368, 497

Edge, A. C. 1990, PhD Thesis, Univ. Leicester

Fabian, A. C., Schwarz, J., \& Forman, W. 1980, MNRAS, 192, 135

Finoguenov, A., \& Jones, C. 2002, ApJ, 574, 74

Finoguenov, A., Pietsch, W., Aschenbach, B., \& Miniati, F. 2004, A\&A, 415, 415

Forman, W., Schwarz, J., Jones, C., Liller, W., \& Fabian, A. C. 1979, ApJ, 234, L27

Machacek, M. E., Jones, C., \& Forman, W. R. 2004, ApJ, 610, 183

Matsushita, K. et al. 1994, ApJL, 436, 41

Maughan, B. J., Ellis, S. C., Jones, L. R., Mason, K. O., Crdova, F. A., \& Priedhorsky, W. 2006, ApJ, 640, 219

Mei, S., et al. 2007, ApJ, 655, 144

Mihos, C., Harding, P., Feldmeier, J., \& Morrison, H. 2005; ApJL, 631,41
Navarro, J. S., Frenk, C. S., \& White, S. D. M. 1995, MNRAS, 275, 720

Neilsen, E. H., \& Tsvetanov, Z. I. 2000, ApJ, 536, 255

Neto et al. 2007, MNRAS, 381, 1450

Nulsen, P. E. J. 1982, MNRAS, 198, 1007

Nulsen, P. E. \& Carter, D. 1987, MNRAS, 225, 939

O'Sullivan, E., Vrtilek, J. M., Kempner, J. C., David, L. P., \& Houck, J. C. 2005, MNRAS, 357, 1134

Rangarajan, F. V. N., White, D. A., Ebeling, H., \& Fabian, A. C. 1995, MNRAS, 277, 1047

Sarazin, C. L., Kundu, A., Irwin, J. A., Sivakoff, G. R., Blanton, E. L., \& Randall, S. W. 2003, ApJ, 595, 743

Schekochinin, A. A., Cowley, S. C., R. M. Kulsrud R. M., Rosin, M. S., \& Heinemann, T. 2007, preprint (astro-ph/0709.3828)

Schindler, S., Binggeli, B., \& Böhringer, H. 1999, A\&A, 343, 420

Smith, R. J., Lucey, J. R., Hudson, M. J., Schlegel, D.J., \& Davies. R. L. 2000, MNRAS, 313, 469

Takano, S., Awaki, H., Koyama, K., Kunieda, H., Tawara, Y. 1989, Nautre, 340, 289

Takeda, H., Nulsen, P. E. J., \& Fabian, A. C. 1984, MNRAS, 208, 261

Vollmer, B., Braine, J., Combes, F., Sofue, Y. 2005, A\&A, 441, 473

White, D. A., Fabian, A. C., Forman, W., Jones, C., \& Stern, C. 1991, ApJ, 375, 35 
TABLE 1

Observation Details

\begin{tabular}{lcccc}
\hline \hline Obs ID & Date Obs & Target & Active CCDs & $\begin{array}{c}\text { Cleaned Exposure }^{\mathrm{a}} \\
(\mathrm{ksec})\end{array}$ \\
\hline 318 & & M86 & S3, S2, I2, I3 & 12.964 \\
803 & $2000-04-07$ & M84 & S3, S2, I2, I3 & 26.699 \\
963 & $2000-05-19$ & M86 & S3, S1, S2, I2, I3 & 13.220 \\
1619 & $2000-04-07$ & NG3, S2, I2, I3 & 19.705 \\
2882 & $2001-06-08$ & NG44838 & S3, S2, I2, I3 & 24.891 \\
5908 & $2002-01-29$ & ME of M86 & S3, S1, S2, I2, I3 & 36.299 \\
5912 & $2005-05-01$ & I0, I1, I2, I3, S2 & 31.370 \\
5913 & $2005-03-09$ & T0, I1, I2, I3, S2 & 34.744 \\
6131 & $2005-03-19$ & Tail Tip & & 37.855 \\
& $2005-11-07$ & & & \\
\hline
\end{tabular}

a Total cleaned exposure is $\sim 238$ ksec. 
TABLE 2

Spectral Fits ${ }^{\mathrm{a}}$

\begin{tabular}{|c|c|c|c|c|c|}
\hline Region \# & $\begin{array}{c}k T \\
(\mathrm{keV})\end{array}$ & $\begin{array}{l}\text { Abund. } \\
\text { (solar) }\end{array}$ & $\Gamma$ & $\chi^{2} /$ dof & Net Cnts. \\
\hline 1 & $0.724_{-0.014}^{+0.013} / /(2.4)$ & $0.42_{-0.13}^{+1.55}$ & $1.71_{-0.68}^{+0.51}$ & $108 / 91=1.18$ & 6235 \\
\hline 1 & $0.677_{-0.021}^{+0.025} / 1.291_{-0.263}^{+0.406} /(2.4)$ & $0.64_{-0.18}^{+0.156}$ & $(1.5)$ & $90 / 89=1.01$ & 6235 \\
\hline 2 & $0.697_{-0.033}^{+0.038} /(1.2) /(2.4)$ & $0.31_{-0.10}^{+0.15}$ & & $56 / 57=0.98$ & 3086 \\
\hline 3 & $0.653_{-0.023}^{+0.016} /(1.2) /(2.4)$ & $0.50_{-0.16}^{+0.90}$ & $(1.5)$ & $88 / 83=1.06$ & 4273 \\
\hline 4 & $0.773_{-0.013}^{+0.013} /(1.2) /(2.4)$ & $0.47_{-0.12}^{+0.17}$ & & $109 / 84=1.29$ & 8630 \\
\hline 5 & $0.854_{-0.031}^{+0.026} /(1.2) /(2.4)$ & $0.40_{-0.09}^{+0.19}$ & & $94 / 93=1.01$ & 4461 \\
\hline 6 & $0.849_{-0.043}^{+0.064} /(1.2) /(2.4)$ & $0.31_{-0.10}^{+0.13}$ & & $89 / 88=1.01$ & 5059 \\
\hline 7 & $0.860_{-0.048}^{+0.061} /(1.2) /(2.4)$ & $0.43_{-0.20}^{+0.39}$ & & $87 / 74=1.18$ & 2843 \\
\hline 8 & $0.785_{-0.130}^{+0.074} /(1.2) /(2.4)$ & $0.45_{-0.17}^{+0.42}$ & & $44 / 40=1.09$ & 1749 \\
\hline 9 & $0.924_{-0.285}^{+0.116} /(1.2) /(2.4)$ & $0.50_{-0.27}^{+2.50}$ & & $30 / 29=1.03$ & 1043 \\
\hline 10 & $1.191_{-0.134}^{+0.127} /(2.4)^{\mathrm{b}}$ & $0.42_{-0.25}^{+0.86}$ & & $20 / 25=0.73$ & 739 \\
\hline 11 & $0.865_{-0.047}^{+0.105 / 1.290_{-0.118}^{+0.240} /(2.4)}$ & $0.55_{-0.15}^{+0.27}$ & & $163 / 176=0.92$ & 8795 \\
\hline 12 & $0.871_{-0.025}^{+0.103} / 1.176_{-0.143}^{+0.235} /(2.4)$ & $0.62_{-0.19}^{+0.28}$ & & $240 / 187=1.29$ & 8914 \\
\hline 13 & $0.675_{-0.187}^{+0.111} / 1.256_{-0.232}^{+0.134} / 2.723_{-0.506}^{+0.744}$ & $0.72_{-0.18}^{+0.19}$ & & $343 / 293=1.17$ & 11033 \\
\hline 14 & $0.625_{-0.010}^{+0.008} /(1.2) /(2.4)$ & $1.17_{-0.10}^{+0.78}$ & $1.82_{-0.12}^{+0.11}$ & $459 / 327=1.40$ & 42002 \\
\hline 15 & $0.790_{-0.06}^{+0.055} /(1.2) /(2.4)$ & $0.60_{-0.18}^{+0.48}$ & & $93 / 90=1.03$ & 3666 \\
\hline 16 & $1.355_{-0.065}^{+0.178} /(2.4)^{\mathrm{b}}$ & $0.49_{-0.17}^{+0.38}$ & & $57 / 59=0.96$ & 1746 \\
\hline 17 & $1.091_{-0.159}^{+0.271} / 2.422_{-0.490}^{+3.328 \mathrm{~b}}$ & $0.40_{-0.20}^{+0.41}$ & & $53 / 45=1.18$ & 3054 \\
\hline 18 & $1.085_{-0.297}^{+0.628} / 2.107_{-0.297}^{+? ? ?}$ & $0.22_{-0.12}^{+0.14}$ & & $118 / 120=0.98$ & 4643 \\
\hline 19 & $0.651_{-0.143}^{+0.077} / 1.065_{-0.188}^{+0.197} /(2.4)$ & $0.25_{-0.09}^{+0.121}$ & & $147 / 128=1.15$ & 7325 \\
\hline 20 & $1.319_{-0.225}^{+0.276} /(2.4)^{\mathrm{b}}$ & $0.39_{-0.25}^{+0.78}$ & & $30 / 23=1.29$ & 1087 \\
\hline 21 & $1.023_{-0.048}^{+0.043} /(2.4)^{\mathrm{b}}$ & $0.22_{-0.07}^{+0.10}$ & & $147 / 105=1.39$ & 2912 \\
\hline 22 & $0.881_{-0.042}^{+0.065} / 1.236_{-0.101}^{+0.156} /(2.4)$ & $0.32_{-0.10}^{+0.07}$ & & $77 / 75=1.03$ & 2646 \\
\hline 23 & $1.186_{-0.160}^{+0.217} / 2.712_{-0.537}^{+1.003 \mathrm{~b}}$ & $0.45_{-0.19}^{+0.34}$ & & $214 / 236=0.91$ & 5193 \\
\hline
\end{tabular}

a Each region contained up to three thermal APEC components: a low temperature, a mid-range temperature, and a high temperature. Temperatures are given, in that order, in column 2. The high temperature component models background Virgo ICM emission. Where this component could not be accurately measured its temperature was fixed at $2.4 \mathrm{keV}$ (see text for discussion). Similarly, the mid-range temperature component models M86 halo emission, and where the temperature could not be measured it was fixed at $1.2 \mathrm{keV}$. The abundance for this component was tied to the abundance of the cooler fitted component. A power law component was included when necessary, presumably for unresolved point sources. Galactic absorption was assumed throughout.

b No low temperature component was included.

${ }^{\mathrm{c}}$ Upper bound of $90 \%$ confidence interval could not be determined. 


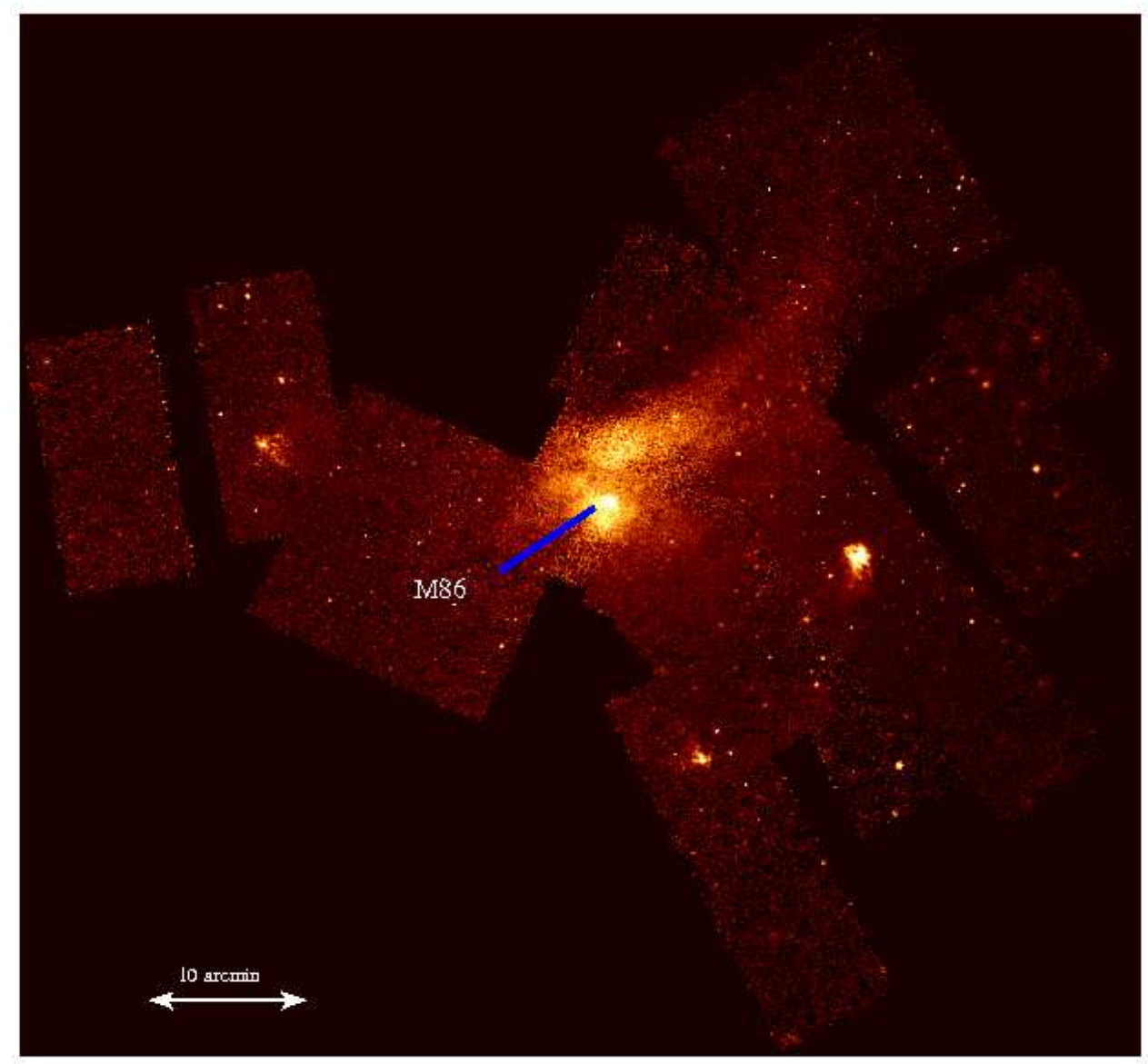

FIG. 1. - Exposure corrected, background subtracted $0.5-2 \mathrm{keV}$ mosaic image of the Chandra observations in the region of M86. The image has been smoothed with a $6^{\prime \prime}$ radius gaussian. For each pointing, regions with less than $10 \%$ of the total exposure for that observation were omitted. Complex structure in the diffuse emission is observed near and in the stripped tail of M86. Directly to the west of M86, M84 is visible, which, aside form showing complicated structure in the core, shows a tail of diffuse emission to the south. Also visible are NGC 4388, to the south of M86 and M84, and NGC 4438, directly east of M86, both of which show structure in their diffuse emission. 


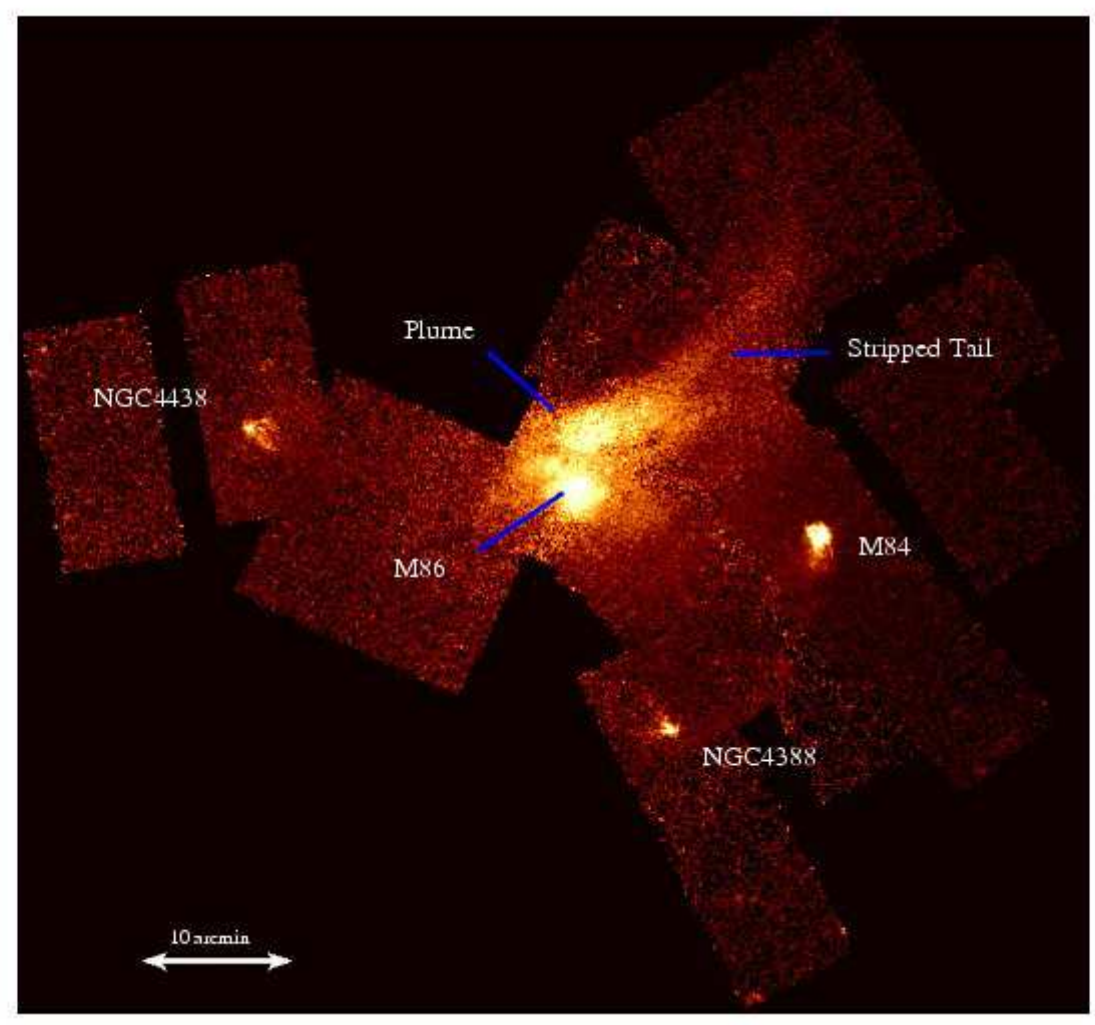

FIG. 2. - Same as Figure 1 but with different scaling and a larger smoothing kernel $\left(9^{\prime \prime}\right)$ to highlight the structure at the low surface brightness end of the M86 tail and to show the faint M84 tail more clearly. Point sources have been removed (see text for details). The main M86 tail, after turning from northwest to north, appears to turn back to the west near the very tip.

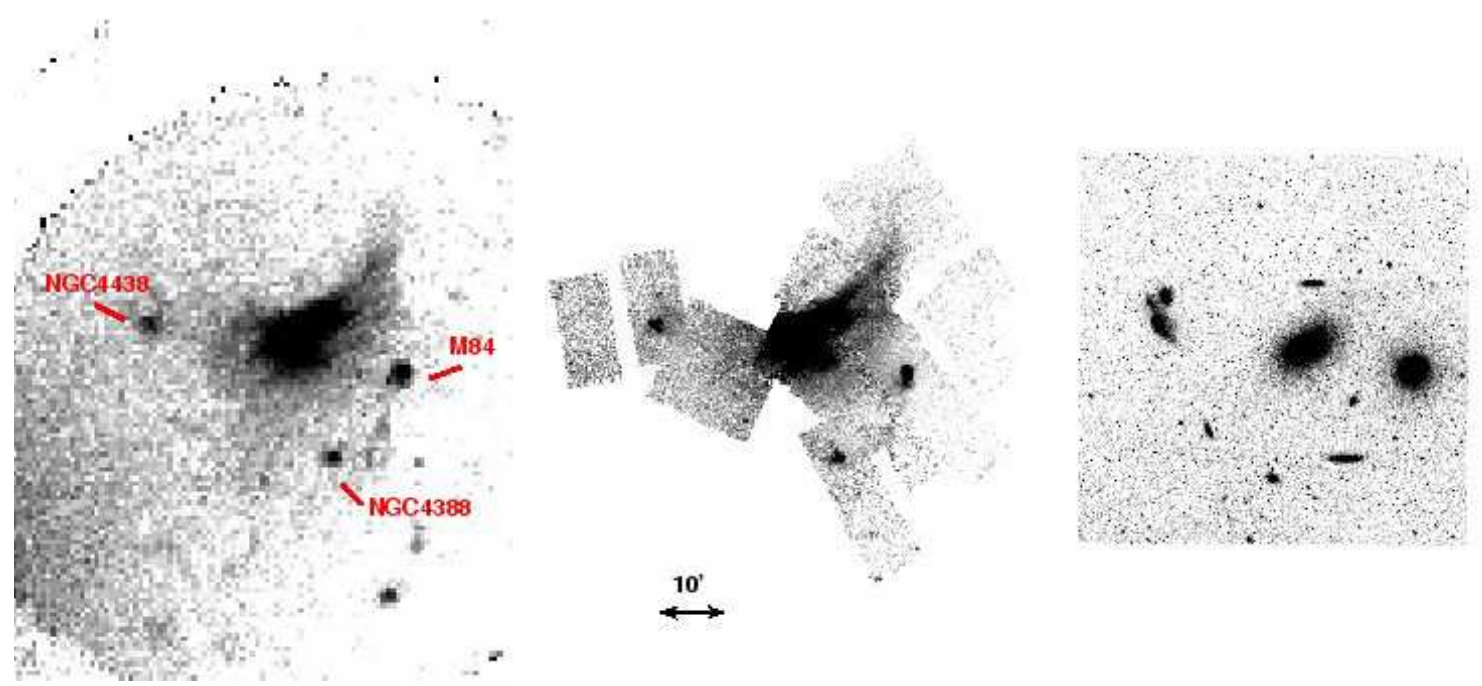

Fig. 3.- (left) ROSAT PSPC view of M86. Diffuse emission from M87/Virgo is clearly visible in the southeast, as is an extended halo of diffuse emission associated with M86. Also shown are the Chandra (center) and DSS (right) images of the same region. 


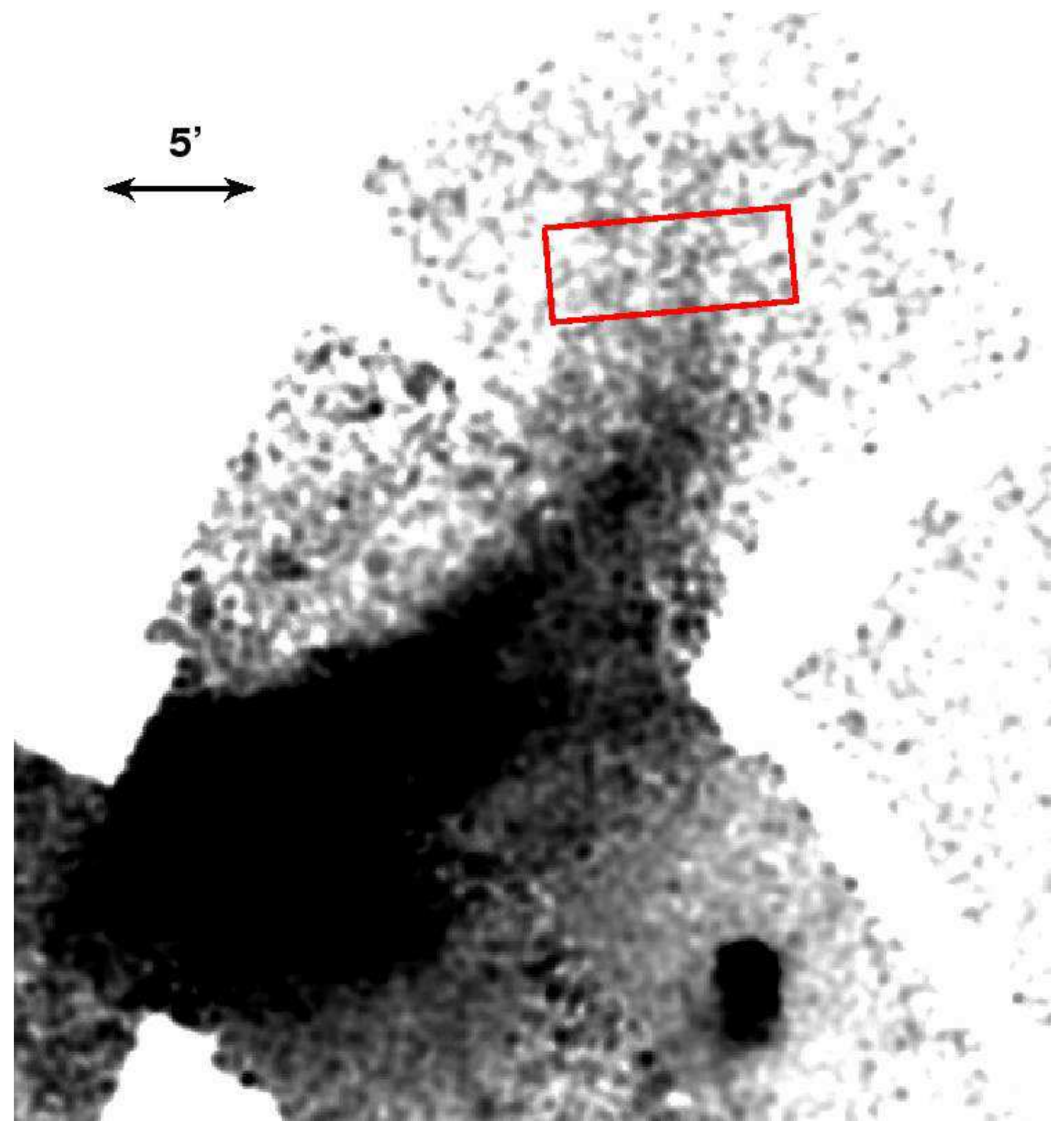

FIG. 4.- Close up view of the long stripped tail, in the $0.5-2 \mathrm{keV}$ band and smoothed with a $16^{\prime \prime}$ radius gaussian. The tail clearly turns from a NW direction to the north, and back directly east at the very tip. Furthermore, there appears to be a faint secondary tail along the northern edge of the faint end of the main tail, which traces its path. The red box indicates the region used to generate the plot in Figure 5 


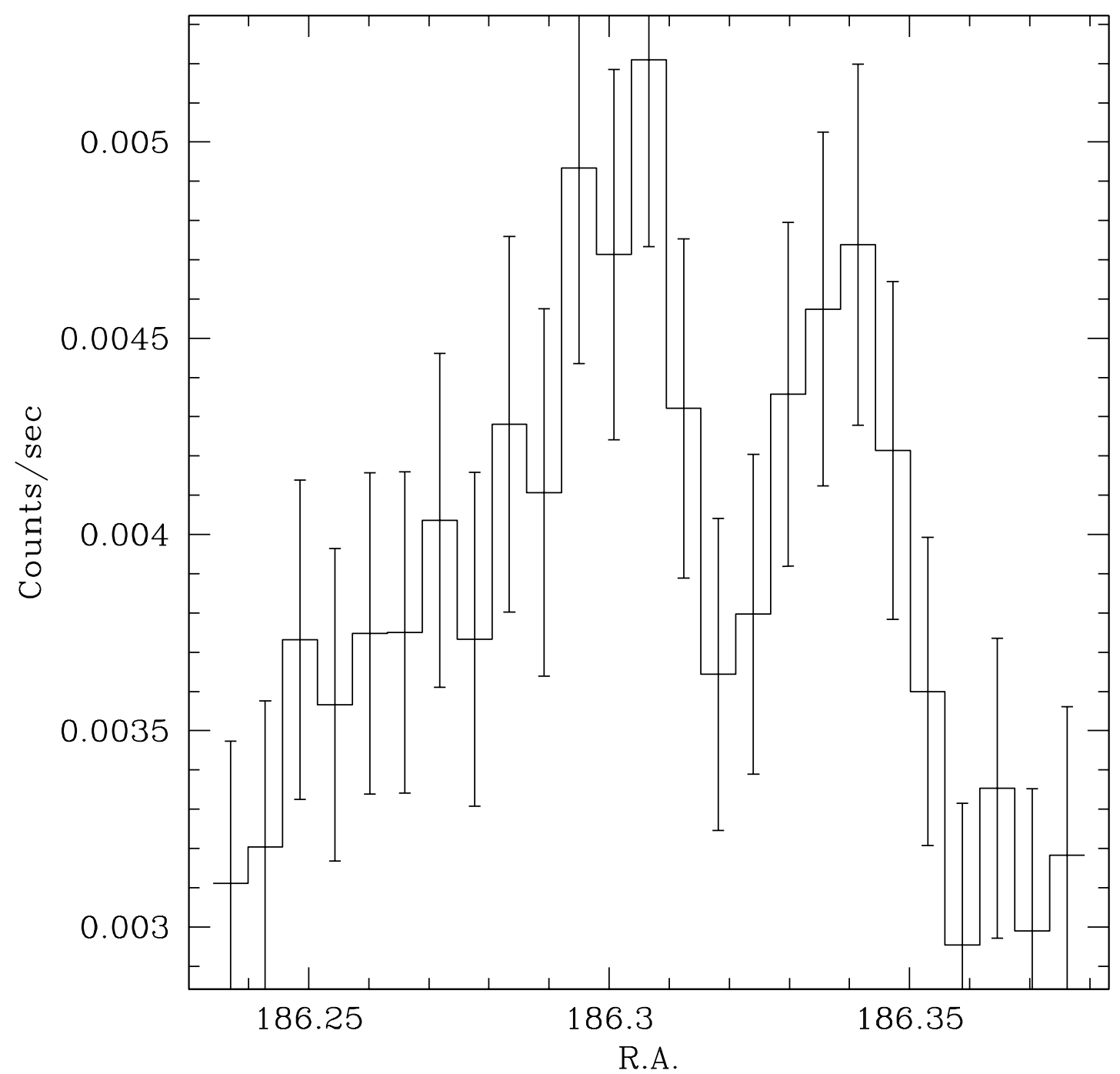

FIG. 5. - Plot of the total count rate in evenly-spaced binned regions taken across the width of the box shown in Figure 4 The $\mathrm{x}$-axis gives the R.A. of each bin on the southern edge of the box. 


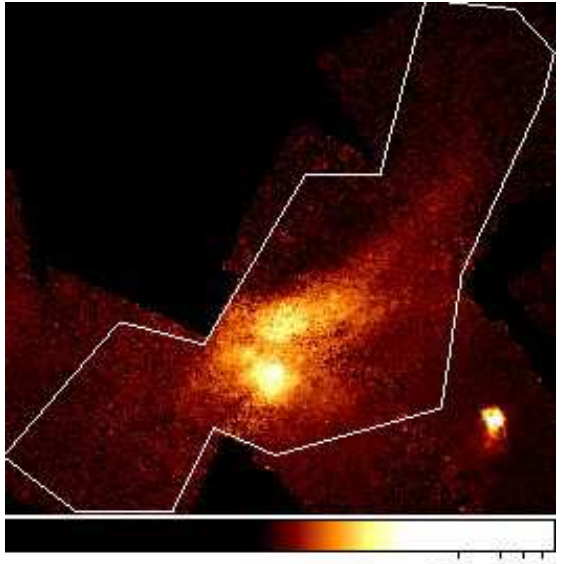

0.00010 .0003

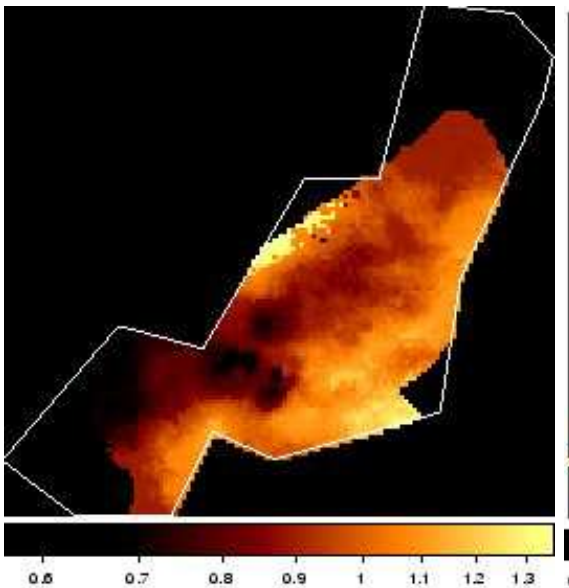

$0.0 \quad 0.7$

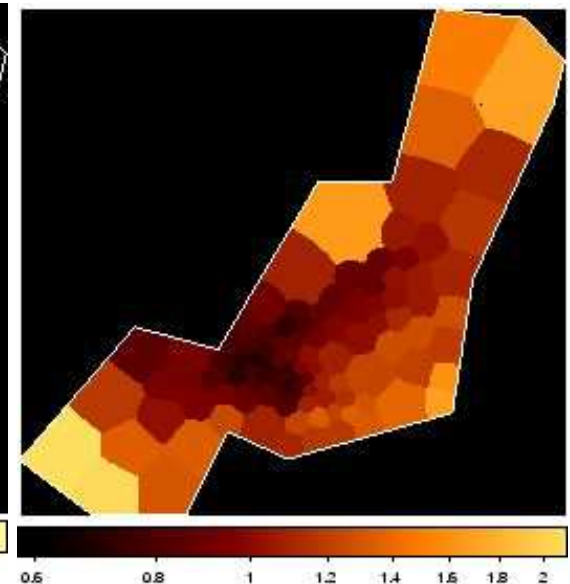

Fig. 6. - Left Panel: A closer view of the central region of Figure 2 with scaling chosen to highlight structure in the central region and nearby diffuse emission. The region used to extract the temperature map is overlain. Center Panel: Derived temperature map of the same region. The contribution from the Virgo ICM has been taken out. Zero-valued pixels indicate areas that required regions larger than the selected maximum area in order to include the minimum net counts (see $\S$ 4.1). Pixels in faint regions which gave an anomalous fitted temperature were also removed. The colorbar under the right panel gives the temperature in keV. Right Panel: Tessellated temperature map. Each bin contains roughly 1100 net counts. The counts in each bin were fit with a single APEC model. The contribution from the Virgo ICM has not been taken out, leading to higher temperatures farther from M86 as compared to the center panel. The size of the bins roughly indicate the size of the extraction region for each pixel in the center panel. 


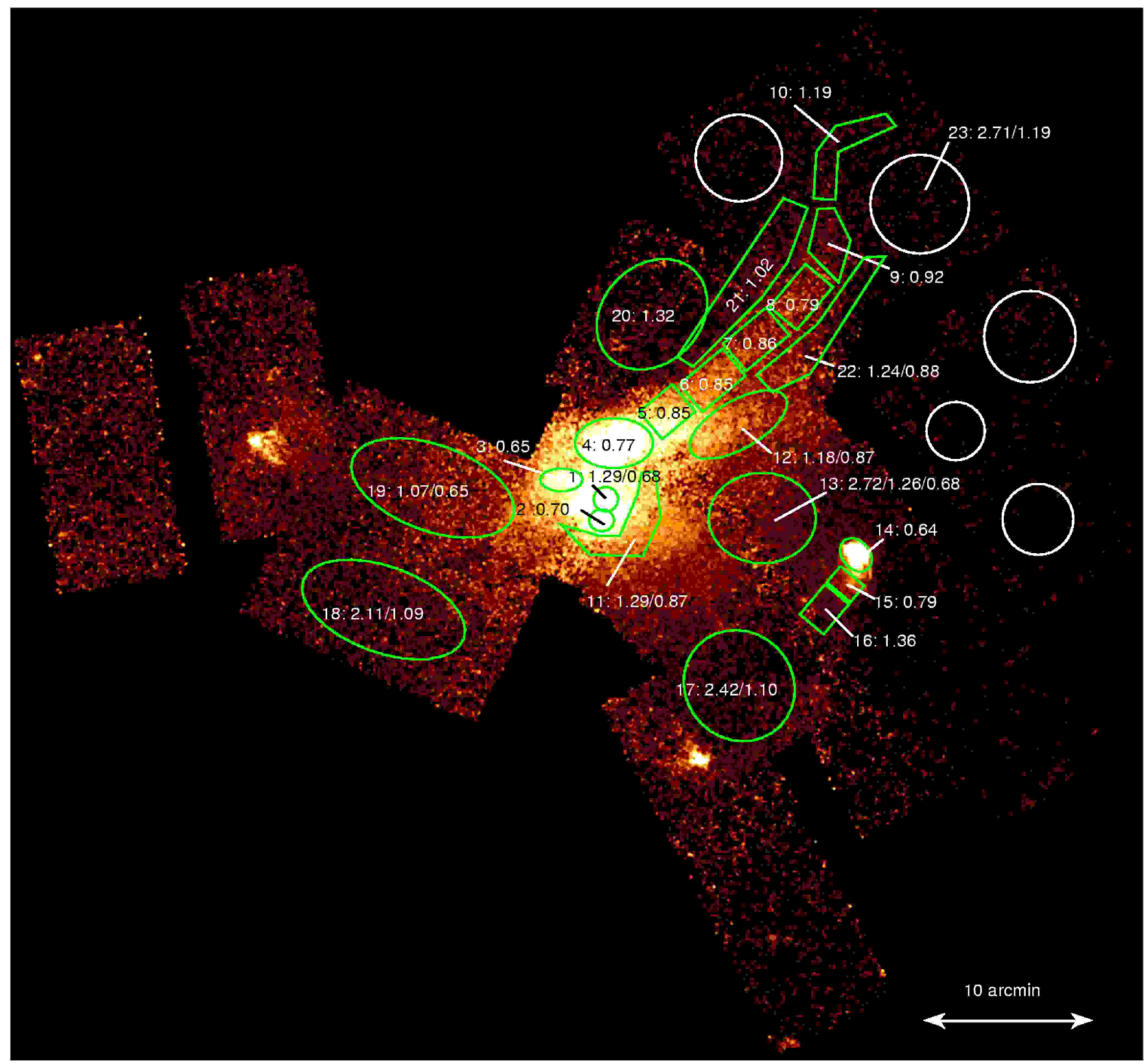

FIG. 7.- Same as Figure 2 but with the regions used for extracting spectra overlain. Single regions for spectral fitting are shown in green, while the regions shown in white are fit as one combined region. Table 2 gives the region number and the best-fit temperature(s). 


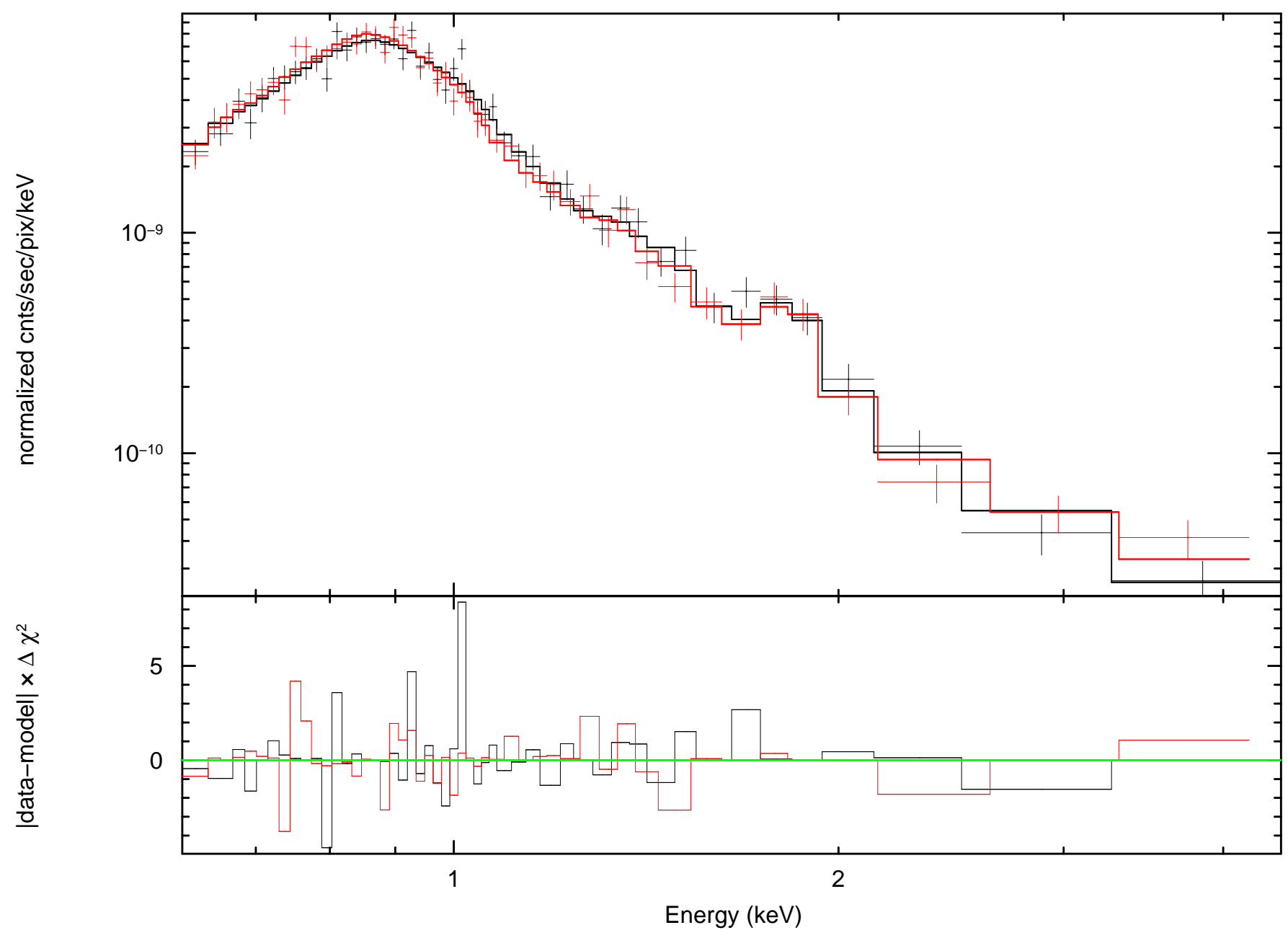

FIG. 8. - Data (crosses) and fitted model (histogram) for the core of M86, R1 in Table 2 The model is a two-temperature thermal plasma with fitted temperatures and abundance (given in the second row of Table2), plus an unresolved power law component with photon index of 1.5 and a fixed thermal plasma component to model emission from the Virgo ICM, all with Galactic absorption. The data are from two S3 observations, OBS-IDs 318 and 963 (see Table 1). 


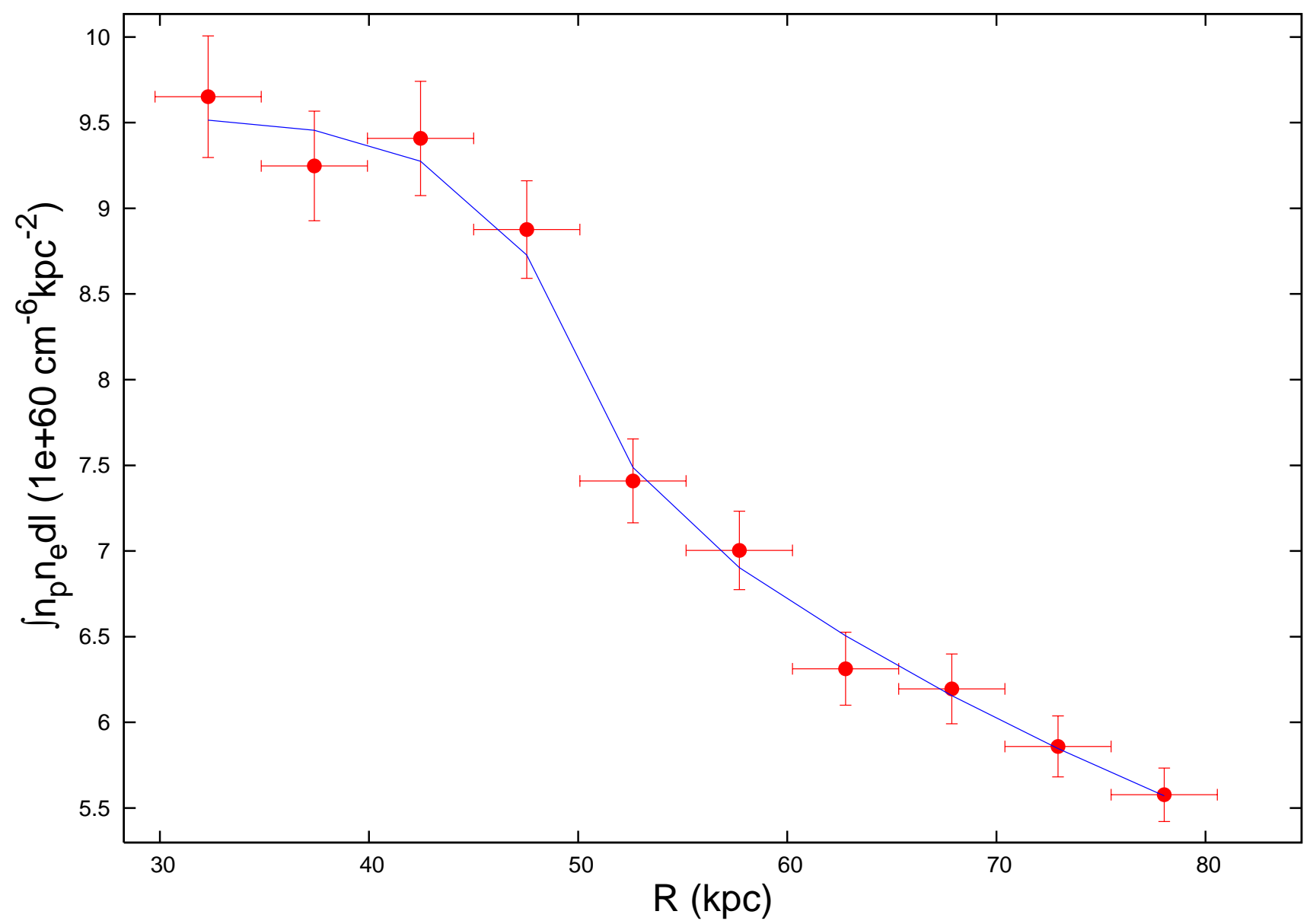

FiG. 9.- Emission measure profile of the northern half of the bright edge seen in the extended group emission southeast of M86 (shown most clearly in the ROSAT image in the left panel of Figure 31. The x-axis gives the radius from the apparent center of curvature defined by the feature. The best fit two power law density jump model is given by the solid line. 


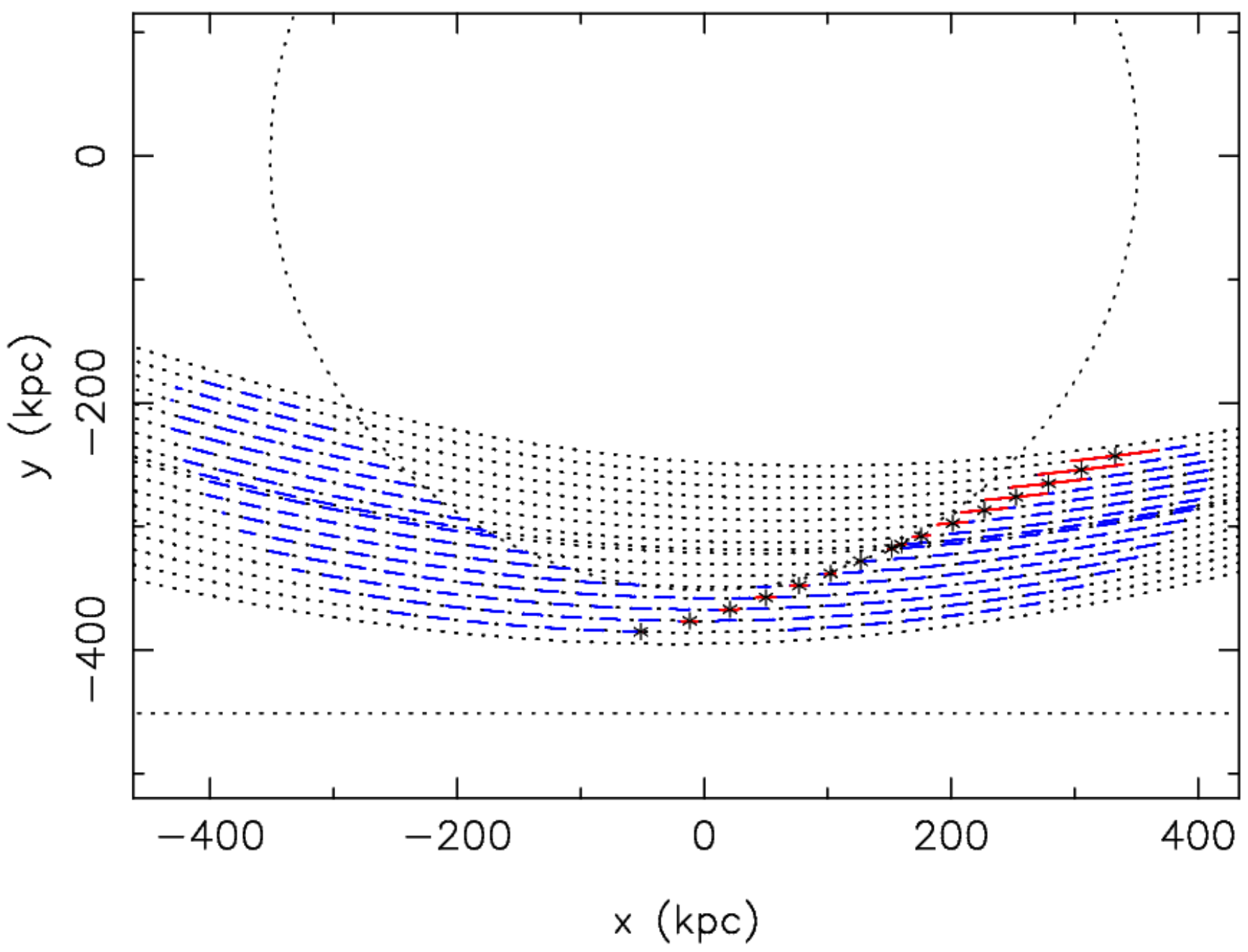

FIG. 10.- Possible marginally bound orbits for M86. Orbits are plotted for inner turning radii in the range $247-395$ kpc. The direction of motion is right to left. The dotted circle shows points $351 \mathrm{kpc}$ from the cluster center. The colored regions (dash-dot and solid lines) show where on each orbit the galaxy could be viewed at a projected distance of $351 \mathrm{kpc}$ from the cluster center, with a line-of-sight speed of $1550 \mathrm{~km} \mathrm{~s}^{-1}$ towards us. Locations on the orbits where the past orbit would also appear to project $100 \mathrm{kpc}$ outside the current position of M86 are shown in red (solid lines; the midpoint of each of these regions is marked with an asterisk). Both regions shrink for orbits with smaller outer turning radii. As plotted, all orbits pass through the virial radius at $x=1300 \mathrm{kpc}$ on the $+x$ axis. 
20

RANDALL ET AL.

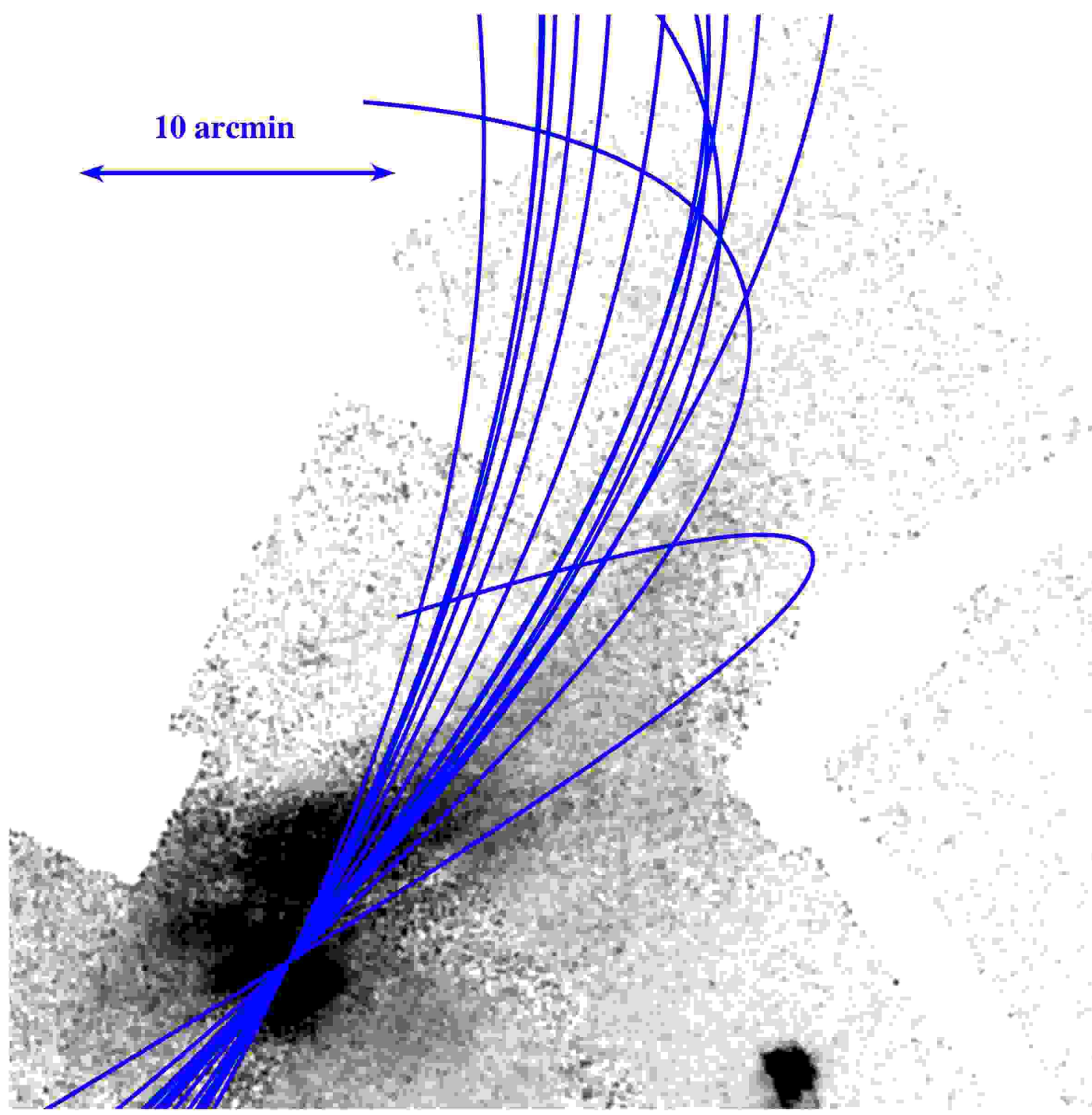

FIG. 11. - Candidate marginally bound orbits for M86. An orbit is shown projected onto the smoothed $0.5-2 \mathrm{keV}$ image of M86 for each of the midpoints of the red regions shown in Figure 10 (marked with an asterisk). Orbits are plotted from the point where they cross the viral radius (sometimes outside the field shown). 


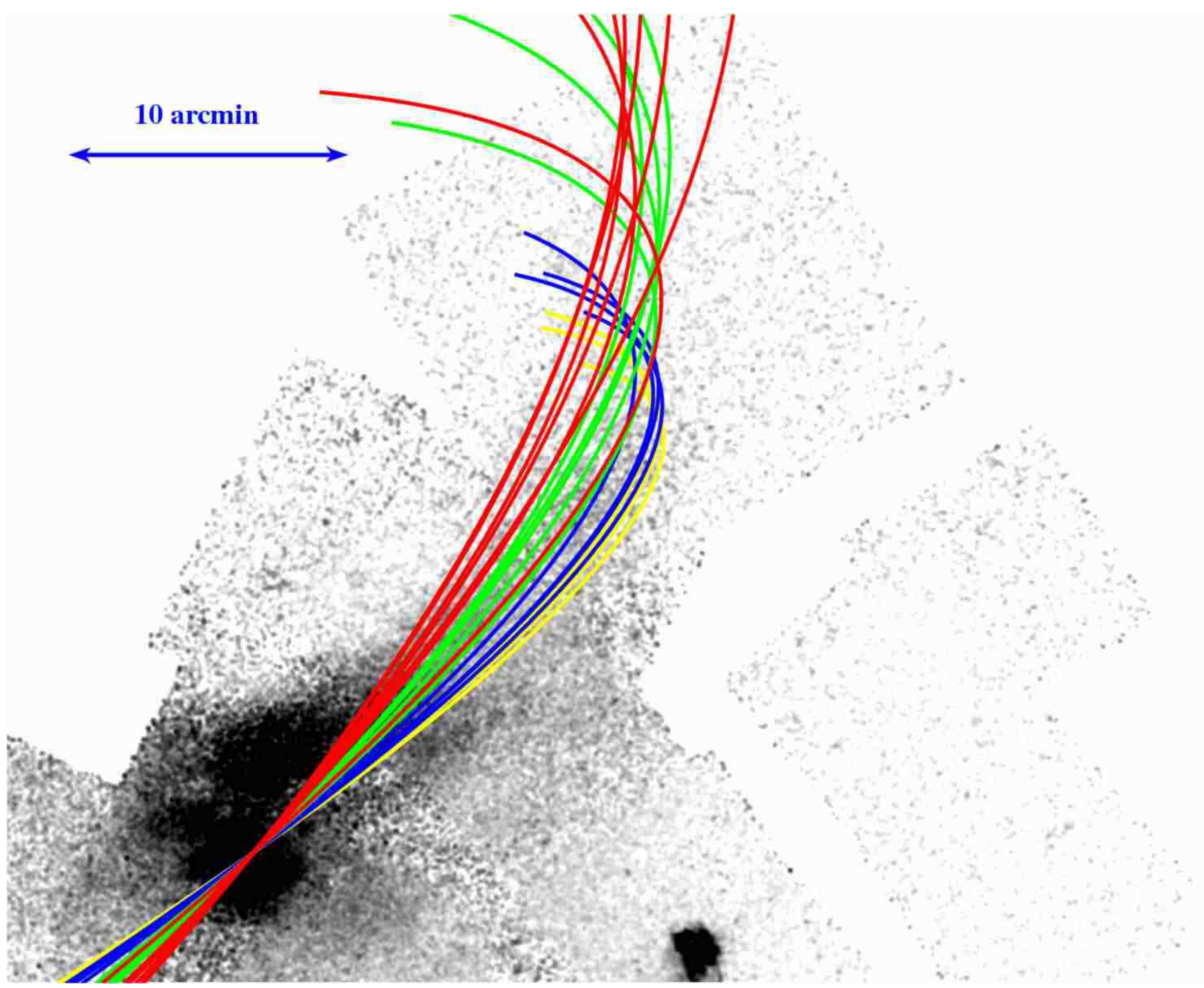

FIG. 12.- Possible orbits for M86. As in Figure11 one representative orbit is shown projected onto the smoothed $0.5-2 \mathrm{keV}$ image of M86 for the remaining plausible orbits. Colors indicate the outer turning radius as follows: $r_{\mathrm{o}}=8.76 \mathrm{Mpc}$, yellow; $r_{\mathrm{o}}=9.11 \mathrm{Mpc}$, blue; $r_{\mathrm{o}}=19.3 \mathrm{Mpc}$, green; $r_{\mathrm{o}}=\infty$, red. 


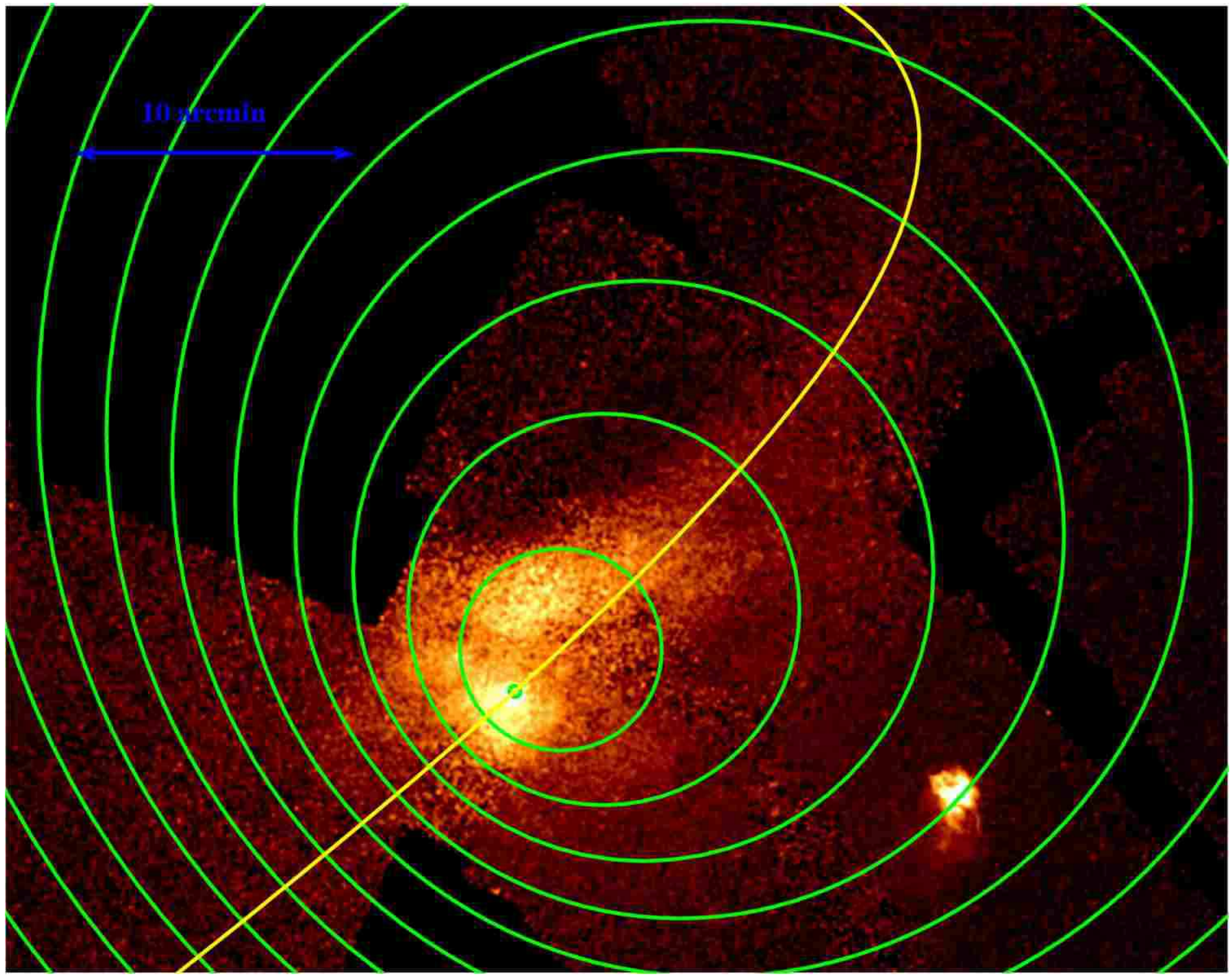

FIG. 13. - Illustration of the Mach cone for M86. Shown in yellow is the marginally bound orbit with $r_{\mathrm{i}}=376 \mathrm{kpc}$, one of the better candidates for the orbit of M86. Centered on the orbit, at equally spaced times, circles are plotted on the plane of the sky with radii $c_{\mathrm{s}} \delta t$, where $\delta t$ is the time before the present when M86 was at that location and $c_{\mathrm{s}}$ is the sound speed $\left(850 \mathrm{~km} \mathrm{~s}^{-1}\right)$. 


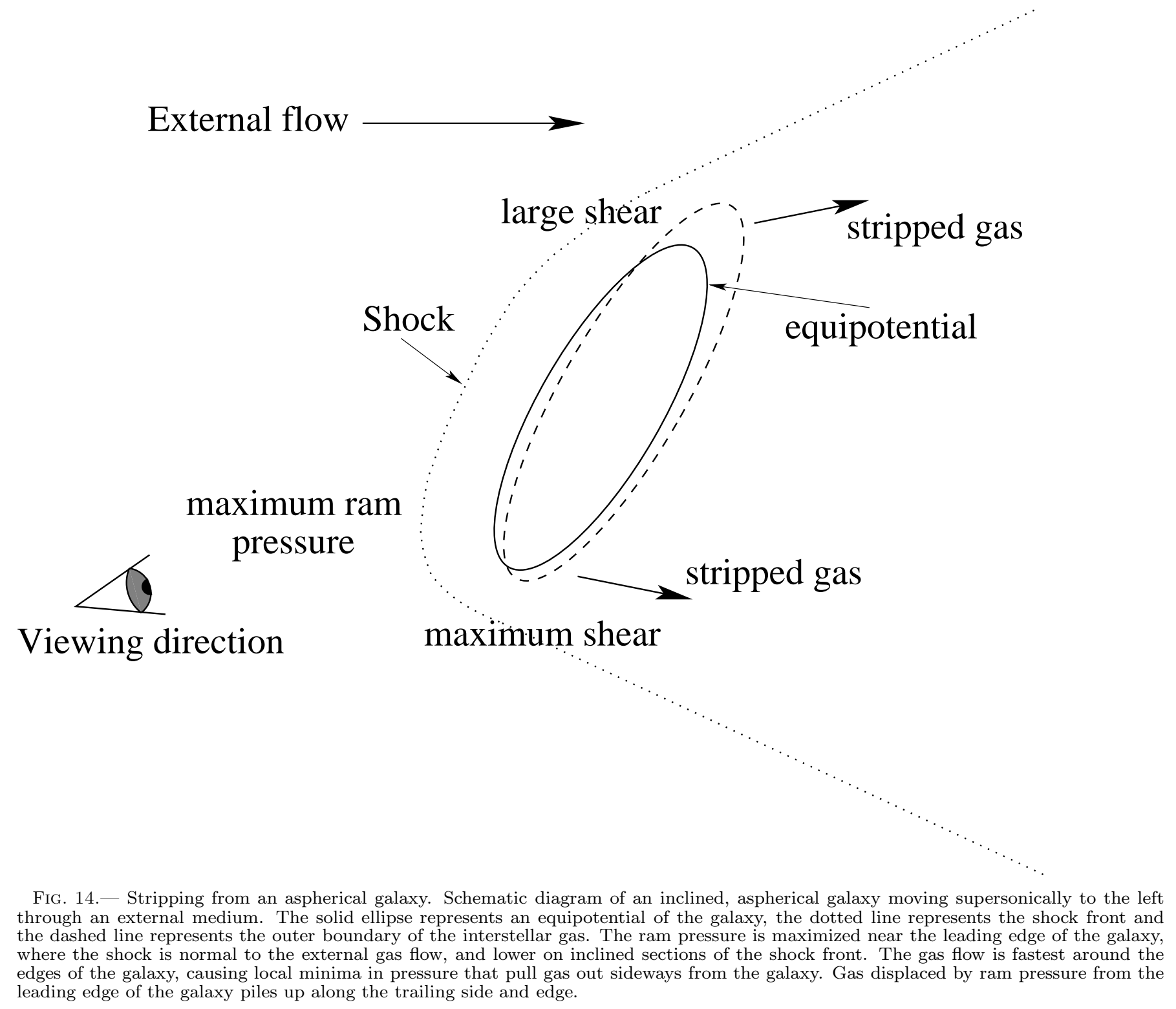




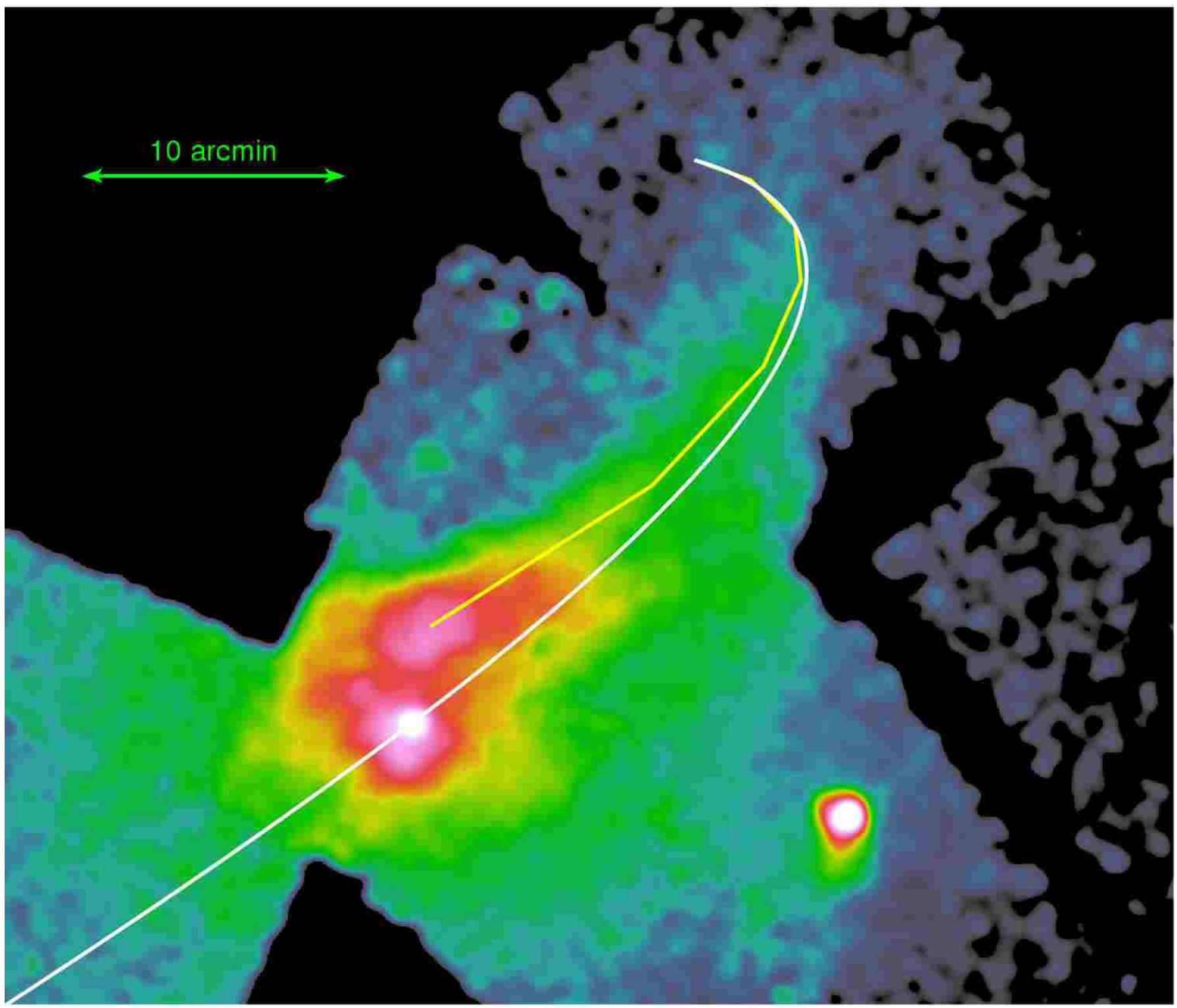

FIG. 15. - Model orbit for ram pressure stripped blob. The orbit of M86 is shown in white and the (coarsely sampled) orbit of the blob is shown in yellow. Note that the path of the blob follows a trail of denser gas. 
APPENDIX

CONSTRAINING M86'S ORBIT

In cylindrical polar coordinates, with the origin at the cluster center and the orbit (instantaneously) in the $x-y$ plane, the position of M86 can be expressed as $\mathbf{r}=r \mathbf{e}_{r}$ and its velocity as $\mathbf{v}=v_{r} \mathbf{e}_{r}+v_{\phi} \mathbf{e}_{\phi}$, where $\mathbf{e}_{r}, \mathbf{e}_{\phi}, \mathbf{e}_{z}$ are the coordinate basis vectors. When M86 is viewed along the unit vector, $\mathbf{n}$, its location projected onto the plane of the sky is $\mathbf{r}-\mathbf{n} \cdot \mathbf{r n}$. Expressing $\mathbf{n}$ in terms of the coordinate vectors as $\mathbf{n}=a^{\prime} \mathbf{e}_{r}+b^{\prime} \mathbf{e}_{\phi}+c^{\prime} \mathbf{e}_{z}$, the projected separation, $s$, of M86 from the cluster center is given by $s^{2}=(\mathbf{r}-\mathbf{n} \cdot \mathbf{r n})^{2}=r^{2}\left(1-a^{\prime 2}\right)$, which requires $a^{\prime}= \pm \sqrt{1-s^{2} / r^{2}}$ (and $r>s)$. Since $\mathbf{n}$ is a unit vector, $a^{\prime 2}+b^{\prime 2}+c^{\prime 2}=1$, further requiring $b^{\prime}= \pm \sqrt{s^{2} / r^{2}-c^{\prime 2}}$. The remaining parameter, $c^{\prime}$, is the cosine of the polar angle of the viewing direction (i.e., the orbit inclination). With the two values of $a^{\prime}$ above, the parametrization $\left(b^{\prime}, c^{\prime}\right)=(\cos \xi, \sin \xi) s / r$, for $0 \leq \xi<2 \pi$, specifies the complete range of viewing directions that project M86 at the observed distance from the cluster center. In this notation, the velocity of M86 along our line-of sight is $v_{\text {los }}=\mathbf{n} \cdot \mathbf{v}=a^{\prime} v_{r}+b^{\prime} v_{\phi}$. For radial orbits with M86 inbound $\left(v_{r}<0\right), v_{\text {los }}=a v_{r}=\sqrt{1-s^{2} / r^{2}} v_{r}<0$. This vanishes for $r \rightarrow s$ (where a radial orbit must be perpendicular to our line-of-sight), while the radial speed, $\left|v_{r}\right|$, decreases with increasing $r$, so that $\left|v_{\text {los }}\right|$ is maximized for some $r>s$.

The inner and outer turning radii, $r_{\mathrm{i}}$ and $r_{\mathrm{o}}$, respectively, are related to the specific energy of an orbit by $E=$ $\left(r_{\mathrm{o}}^{2} \phi_{\mathrm{o}}-r_{\mathrm{i}}^{2} \phi_{\mathrm{i}}\right) /\left(r_{\mathrm{i}}^{2}-r_{\mathrm{o}}^{2}\right)$, where $\phi_{\mathrm{i}}=\phi\left(r_{\mathrm{i}}\right)$ is the gravitational potential at $r_{\mathrm{i}}$ and $\phi_{\mathrm{o}}$ is that at $r_{\mathrm{o}}$. The specific angular momentum of the orbit, $\ell$, is given by $\ell^{2}=2 r_{\mathrm{i}}^{2} r_{\mathrm{o}}^{2}\left(\phi_{\mathrm{o}}-\phi_{\mathrm{i}}\right) /\left(r_{\mathrm{o}}^{2}-r_{\mathrm{i}}^{2}\right)$. If M86 is bound to the Virgo cluster, then, since $r \geq r_{\mathrm{i}}$ and its speed, $v \geq\left|v_{\text {los }}\right|, 0>E=v^{2} / 2+\phi(r) \geq v_{\text {los }}^{2} / 2+\phi_{\mathrm{i}}$, which requires $\phi_{\mathrm{i}}<-v_{\text {los }}^{2} / 2$. Taking $v_{\text {los }}=-1550 \mathrm{~km} \mathrm{~s}^{-1}$, this gives an upper limit on the inner turning radius of $r_{\mathrm{i}}<489 \mathrm{kpc}$. To obtain a lower limit on $r_{\mathrm{o}}$, note that $\partial E / \partial r_{\mathrm{o}}=\left(v_{\mathrm{K}, \mathrm{o}}^{2}-v_{\mathrm{o}}^{2}\right) r_{\mathrm{o}} /\left(r_{\mathrm{o}}^{2}-r_{\mathrm{i}}^{2}\right)$, where the Kepler speed at $r_{\mathrm{o}}$ is given by $v_{\mathrm{K}, \mathrm{o}}^{2}=r d \phi /\left.d r\right|_{r=r_{\mathrm{o}}}$ and the speed at the outer turning radius is $v_{\mathrm{o}}^{2}=\ell^{2} / r_{\mathrm{o}}^{2}=2\left(E-\phi_{\mathrm{o}}\right)$. The net acceleration at the outer turning radius is inward, requiring $v_{\mathrm{o}}<v_{\mathrm{K}, \mathrm{o}}$, so that $E$ is an increasing function of $r_{\mathrm{o}}$. Interchanging $r_{\mathrm{i}}$ and $r_{\mathrm{o}}$ in this argument and noting that the net acceleration is outward at the inner turning radius $\left(v_{\mathrm{K}, \mathrm{i}}<v_{\mathrm{i}}\right)$ shows that $E$ is also an increasing function of $r_{\mathrm{i}}$. Since $d E=\partial E / \partial r_{\mathrm{i}} d r_{\mathrm{i}}+\partial E / \partial r_{\mathrm{o}} d r_{\mathrm{o}}$, for a fixed energy, maximizing $r_{\mathrm{i}}$ minimizes $r_{\mathrm{o}}$. For M86, $v \geq\left|v_{\mathrm{los}}\right|$ and $r \geq s$, so that its energy $E \geq E_{\min }=v_{\text {los }}^{2} / 2+\phi(s)$. To obtain $E=E_{\min }$ with $r_{\mathrm{i}}=s$ requires $r_{\mathrm{o}}=r_{\mathrm{o}, \min } \simeq 3.5 \mathrm{Mpc}$, or $\simeq 2.7$ times the virial radius of the cluster. For values of $r_{\mathrm{i}}$ larger than $s$, the total energy would need to match or exceed $v_{\mathrm{los}}^{2} / 2+\phi_{\mathrm{i}}>E_{\mathrm{min}}$. The value of $r_{\mathrm{o}}$ required to attain this energy is an increasing function of $r_{\mathrm{i}}$, unless the mean density of the cluster increases with radius. Thus, $r_{\mathrm{o}, \text { min }}$ provides a lower limit on the outer turning radius of M86's orbit.

At a fixed point on an orbit, the requirement $v_{\text {los }}=a^{\prime} v_{r}+b^{\prime} v_{\phi}$ determines the possible viewing directions, if any, that could model M86. For each of the two solutions $a^{\prime}= \pm \sqrt{1-s^{2} / r^{2}}$, the requirement $v_{\text {los }}=a^{\prime} v_{r}+b^{\prime} v_{\phi}$ gives $b^{\prime}=\left(v_{\text {los }}-a^{\prime} v_{r}\right) / v_{\phi}$. If either of these gives $b^{\prime}$ in the range $[-s / r, s / r]$, then it corresponds to two possible viewing directions, with $c^{\prime}= \pm \sqrt{s^{2} / r^{2}-b^{\prime 2}}$ (symmetrically placed above and below the plane of the orbit). Thus, for each point on an orbit, there can be zero, two, or four potential viewing directions that would place M86 at the observed separation from the cluster center and give it the observed line-of-sight velocity. Figure 10 shows a range of marginally bound orbits $\left(r_{\mathrm{o}}=\infty\right)$, with regions that meet these conditions marked in color. Since $\left|v_{\text {los }}\right|<v_{\text {los, } \max }=\sqrt{1-s^{2} / r^{2}}\left|v_{r}\right|+s / r\left|v_{\phi}\right|$, which is an increasing function of $r_{\mathrm{o}}$ for fixed $r$ and $r_{\mathrm{i}}$, the range of an orbit where these conditions are met shrinks as $r_{\mathrm{o}}$ decreases, i.e., as the orbit becomes more tightly bound.

If the trail of stripped gas lies along the orbit of M86, then the past orbit must project away from the cluster center at least as far as the gas tail extends. From above, $(\mathbf{r}-\mathbf{n} \cdot \mathbf{r n}) / s$ is a unit vector in the plane of the sky that points from the cluster center towards M86. For each potential location of M86 on an orbit, we have computed $d\left(\mathbf{r}^{\prime}\right)=\mathbf{r}^{\prime} \cdot(\mathbf{r}-\mathbf{n} \cdot \mathbf{r n}) / s$ for values of $\mathbf{r}^{\prime}$ ranging over the past orbit. If stripping is to account for the location of the gas tail, $d\left(\mathbf{r}^{\prime}\right)$ must increase initially as $\mathbf{r}^{\prime}$ moves backward along the orbit and the maximum value of $d\left(\mathbf{r}^{\prime}\right)-s$ must exceed the distance, $\sim 100 \mathrm{kpc}$, that the tail projects beyond M86 in the direction away from the cluster center. Locations on the orbits where the line-of-sight speed can attain $-1550 \mathrm{~km} \mathrm{~s}^{-1}$ and these conditions are also met are shown in red in Figure 10. 